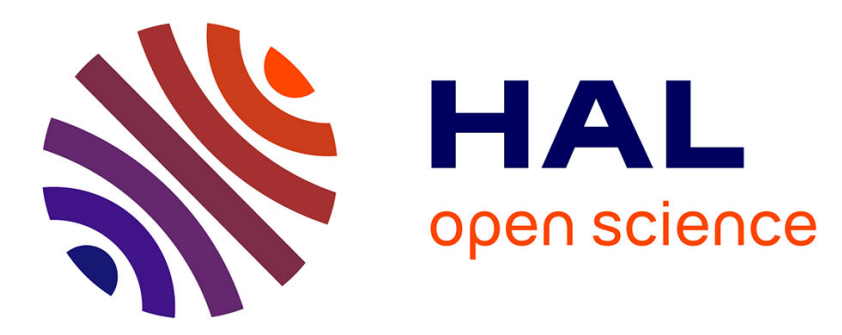

\title{
Supramodal processing optimizes visual perceptual learning and plasticity
}

Nicolas Zilber, Philippe Ciuciu, Alexandre Gramfort, Leila Azizi, Virginie van Wassenhove

\section{- To cite this version:}

Nicolas Zilber, Philippe Ciuciu, Alexandre Gramfort, Leila Azizi, Virginie van Wassenhove. Supramodal processing optimizes visual perceptual learning and plasticity. NeuroImage, 2014, 93, pp.32 - 46. 10.1016/j.neuroimage.2014.02.017 . hal-01084251

\section{HAL Id: hal-01084251 \\ https://inria.hal.science/hal-01084251}

Submitted on 18 Nov 2014

HAL is a multi-disciplinary open access archive for the deposit and dissemination of scientific research documents, whether they are published or not. The documents may come from teaching and research institutions in France or abroad, or from public or private research centers.
L'archive ouverte pluridisciplinaire HAL, est destinée au dépôt et à la diffusion de documents scientifiques de niveau recherche, publiés ou non, émanant des établissements d'enseignement et de recherche français ou étrangers, des laboratoires publics ou privés.

\section{다)(1) $\$$}

Distributed under a Creative Commons Attribution - NonCommercial| 4.0 International 


\section{Supramodal processing optimizes visual perceptual learning and plasticity}

Nicolas Zilber $^{\mathrm{a}, \mathrm{b}}$, Philippe Ciuciu ${ }^{\mathrm{a}, \mathrm{b}}$, Alexandre Gramfort ${ }^{\mathrm{a}, \mathrm{b}, \mathrm{c}}$, Leila Azizi ${ }^{\mathrm{a}, \mathrm{d}, \mathrm{e}}$, Virginie van

Wassenhove $\mathrm{a}^{\mathrm{a}, \mathrm{d}, \mathrm{e}, 1}$

\section{Author affiliations:}

${ }^{\text {a }}$ CEA, DSV/I2BM, NeuroSpin Center, F-91191 Gif-sur-Yvette, France

${ }^{\mathrm{b}}$ INRIA, Parietal team, Saclay, France

${ }^{\mathrm{c}}$ Institut Mines-Télécom, Télécom ParisTech, CNRS LTCI, 75014 Paris, France

${ }^{d}$ INSERM, U992, Cognitive Neuroimaging Unit, F-91191 Gif-sur-Yvette, France

${ }^{\mathrm{e}}$ Univ Paris-Sud, Cognitive Neuroimaging Unit, F-91191 Gif-sur-Yvette, France

\section{Corresponding author:}

${ }^{1}$ CEA.DSV.I ${ }^{2}$ BM.NeuroSpin - INSERM Cognitive Neuroimaging Unit

NeuroSpin MEG, Brain Dynamics group

Bât 145 Point Courrier 156 F-91191 Gif-sur-Yvette France

Phone: +33 (0)1 69081667

Virginie.van.Wassenhove@gmail.com

KEYWORDS: MEG, multisensory, audition, coherence, dual-stream, sensory substitution device. 


\section{ABSTRACT}

Multisensory interactions are ubiquitous in cortex and it has been suggested that sensory cortices may be supramodal i.e. capable of functional selectivity irrespective of the sensory modality of inputs (Pascual-Leone and Hamilton, 2001; Ricciardi and Pietrini, 2011; Voss and Zatorre, 2012; Renier et al., 2013). Here, we asked whether learning to discriminate visual coherence could benefit from supramodal processing. To this end, three groups of participants were briefly trained to discriminate which of a red or green intermixed population of random-dot-kinematograms (RDKs) was most coherent in a visual display while being recorded with magnetoencephalography (MEG). During training, participants heard no sound $(\mathrm{V})$, congruent acoustic textures (AV) or auditory noise (AVn); importantly, congruent acoustic textures shared the temporal statistics - i.e. coherence - of visual RDKs. After training, the AV group significantly outperformed participants trained in $\mathrm{V}$ and $\mathrm{AVn}$ although they were not aware of their progress. In pre- and post-training blocks, all participants were tested without sound and with the same set of RDKs. When contrasting MEG data collected in these experimental blocks, selective differences were observed in the dynamic pattern and the cortical loci responsive to visual RDKs. First and common to all three groups, vlPFC showed selectivity to the learned coherence levels whereas selectivity in visual motion area hMT+ was only seen for the AV group. Second and solely for the AV group, activity in multisensory cortices (mSTS, pSTS) correlated with post-training performances; additionally, the latencies of these effects suggested feedback from vlPFC to hMT+ possibly mediated by temporal cortices in AV and AVn groups. Altogether, we interpret our results in the context of the Reverse Hierarchy Theory of learning (Ahissar and Hochstein, 2004) in which supramodal processing optimizes visual perceptual learning by capitalizing on sensory-invariant representations - here, global coherence levels across sensory modalities. 


\section{INTRODUCTION}

Increasing evidence for multisensory integration throughout cortex has challenged the view that sensory systems are strictly independent (Driver and Spence, 2000; Ghazanfar and Schroeder, 2006), questioning in turn the innate specialization of sensory cortices. For instance early in development, auditory neurons can respond to light patches when rewired to receive visual information (Roe et al., 1990; Mao et al., 2011) and cooling specific parts of auditory cortex in deafened cats selectively perturbs the detection of visual motion and localization (Lomber et al., 2010). In congenitally blind humans, the cortical area hMT+ responsive to visual motion (human homolog of MT/V5 in monkeys) is recycled for auditory or tactile processing (Poirier et al., 2005; Ricciardi et al., 2007; Bedny et al., 2010; Watkins et al., 2013) and the ventral and dorsal visual processing streams develop their functional specificity even when deprived of direct visual experience (Striem-Amit et al., 2012). Consistent with these observations, the "metamodal theory" (Pascual-Leone and Hamilton, 2001) and the "supramodal hypothesis" (Ricciardi and Pietrini, 2011; Voss and Zatorre, 2012) have suggested that some cortical areas may be naturally capable of functional selectivity irrespective of the sensory modality of inputs, hence of functional recycling. However, several questions have been raised (Bavelier and Hirshorn, 2010) among which: is functional recycling a consequence of sensory deprivation during a sensitive period or does it rely on pre-existing supramodal computational capabilities (Bedny et al., 2010; Morrone, 2010; Dormal and Collignon, 2011; Renier et al., 2013)? 
In order to specifically address this issue, we trained non-sensory impaired individuals on a difficult and novel visual task and asked whether visual learning and plasticity would benefit from matched audiovisual stimulation. For this, three different groups of twelve individuals were recorded with magnetoencephalography (MEG) while they performed a visual discrimination task. MEG blocks consisted of a pre-training, a 20 minutes individualized training and a post-training (Figure 1A). It should be stressed that a short-training (20 minutes total) was used in all three training conditions; hence, we were interested in the possible effects of multisensory learning within a very short time period which may not match those obtained over days of training (e.g. (Shams and Seitz, 2008)). The main task consisted in determining which of a red or green intermixed population of random-dot-kinematograms (RDKs) was most coherent in the visual display (Figure 1B); hence, this task implicated motion-color binding (what/where integration) and visual motion coherence discrimination. The pre- and post-training sessions were exclusively visual and tested the same RDK coherence levels for all three groups of participants; on the other hand, training sessions were individualized with regards to the RDK coherence levels and the training context. Specifically, participants could be trained in silence (V), with correlated acoustic textures (AV), or with auditory noise (AVn, control group). In the AV group, AV stimuli sharing redundant temporal regularities were designed using auditory analogs of visual RDKs i.e. acoustic textures (Overath et al., 2010) (Figure 1C). In the control AVn group, the auditory stimulus was filtered noise. Both AV and AVn groups were told to neglect the sounds played in the background; in the AV group and unbeknownst to participants, the coherence of acoustic textures matched that of the target RDK (see Materials and Methods, Figure 1C).

Crucially, and for all participants, the RDK coherence levels were the sole criterion enabling to properly perform the task: first, the direction taken by the coherent dots was randomized 
across trials and orthogonal to the coherence level; second, acoustic textures could not inform on the color of the most coherent RDK albeit shared their dynamics; third, acoustic textures were kept minimally accessible to participants' awareness. Additionally, all reported results exclusively focus on the comparison of pre- and post-training data in which no acoustic information was delivered to any of the participants (Figure 1A): hence, we do not address the issue of multisensory integration per se (which takes place a priori during the training blocks) and instead focus on the effect of participants' training history on perceptual learning and cortical plasticity.
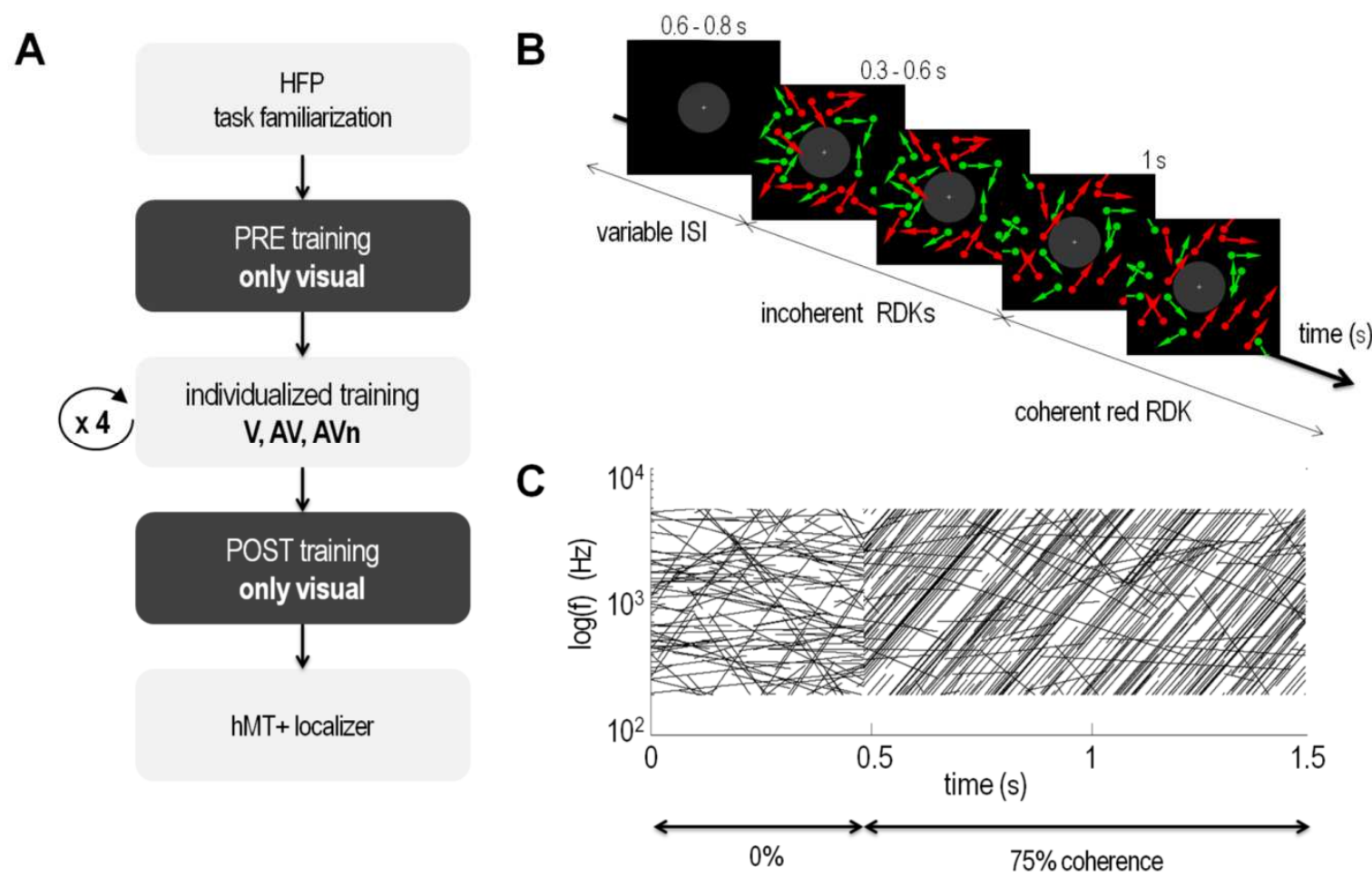

Figure 1. Experimental design and stimuli. Panel A: An MEG session for one individual was composed of several blocks: first, the luminance of the red and green Random Dot Kinematograms (RDKs) was calibrated using Heterochromatic Flicker Photometry (HFP). Luminance calibration was followed by a few familiarization trials to the task during which participants received feedback. In the pre-training block, all participants were presented with stimuli that were solely visual. The pre-training data established the set of coherence levels for the training session based on an individual's coherence discrimination threshold. In the following four training blocks, participants were trained with four levels of RDK coherence. The four training blocks lasted 20 minutes and were without feedback. The training could be visual only (V), audiovisual using acoustic textures (AV) or audiovisual using acoustic noise (AVn). In the post-training block, each individual's coherence discrimination threshold was established with visual stimulation only. In the last block, a localizer provided an independent 
means to source localize the Human motion area hMT+ using combined functional MEG localizer data and anatomical MRI (see Methods). Panel B: An experimental trial consisted in the presentation of a fixation cross followed by the appearance of two intermixed and incoherent RDKs (red and green populations). After 0.3 to 0.6 s, one of the two RDKs became more coherent than the other: the red RDK is here illustrated as the most coherent. Participants were asked to indicate which of the red or green population was most coherent irrespective of the direction of motion. Panel C: Sample spectrogram in $\log$ (frequency) as a function of time depicting an acoustic texture. By analogy to a visual RDK, the level of coherence in an acoustic texture was defined as the number of frequency ramps sharing the same slope in a given frequency range. Here, the spectrogram illustrates an incoherent acoustic texture lasting $500 \mathrm{~ms}$ followed by a 75\% coherent acoustic texture lasting $1 \mathrm{~s}$. Three groups of participants underwent three types of training. During V training, participants were solely provided with visual stimuli; during AV training, an acoustic texture was paired with the most coherent RDK population and the acoustic transition from incoherent to coherent was synchronized with the visual transition in the RDKs; during the AVn control training, the sound was a white noise unrelated to the visual RDKS. Inter-stimulus intervals were randomly drawn from 0.6 to $0.8 \mathrm{~s}$. $\min =$ minutes; $\mathrm{s}=$ seconds; $\mathrm{ms}=$ milliseconds.

\section{MATERIALS AND METHODS}

\subsection{Participants}

All participants were right-handed, had normal hearing and normal or corrected-to-normal vision. Participants' ages ranged between 18 and 28 years old (mean age: $22.1 \pm 2.2$ s.d.). Prior to the study, participants were randomly split into three training groups, namely: an only visual training group ( $\mathrm{V}, \mathrm{n}=12,4$ females), an audiovisual training group using acoustic textures (AV group, $\mathrm{n}=12,6$ females) or using acoustic noise (AVn which is also a control group, $n=12,6$ females). Before the experiment, all participants provided a written informed consent in accordance with the Declaration of Helsinki (2008) and the local Ethics Committee on Human Research at NeuroSpin (Gif-sur-Yvette, France).

\subsection{Experimental design}


The magnetoencephalography (MEG) experiment was conducted in a darkened soundproof magnetic-shielded room (MSR). Participants were seated in upright position under the MEG dewar and faced a projection screen placed $90 \mathrm{~cm}$ away. We used a Panasonic DLP projector (model PT-D7700E-K, Panasonic Inc, Kadoma, Japan) with a refresh rate of $60 \mathrm{~Hz}$. The sound pressure level was set at a comfortable hearing level $(\sim 65 \mathrm{~dB})$ for all participants. Participants were explained the task and stayed in contact at all times with the experimenter via a microphone and a video camera. Stimuli (see videos S1 and S2 for AV and AVn, respectively) were designed using Matlab (R2010a, Mathworks Inc.) with Psychtoolbox-3 (Pelli, 1997) on a PC (Windows XP).

The experiment consisted of eight consecutive blocks (Figure1a). First, Heterochromatic Flicker Photometry (HFP) was used for luminance calibration of the red and green Random Dots Kinematograms (RDKs) on a per individual basis. Second, participants were familiarized with the task and the stimuli: they were presented with 16 easy trials displaying two intermixed Random Dot Kinematograms (RDKs). One of the two RDKs was 100\% coherent - i.e. all dots took the same direction of motion. Participants were asked to report the color of the most coherent RDK by pressing a "green" (green RDK) or a "red" (red RDK) button. During this short familiarization block, participants were provided with feedback on their performance. No other feedback was provided in the remainder of the experimental session.

After this familiarization block, participants underwent a 12 minutes pre-training session, hereafter referred to as PRE training. As in the familiarization block, participants were asked to report as accurately as possible which of the two RDKs was most coherent by selecting the “green” (green RDK) or "red” button (red RDK). This task was subsequently used in all experimental blocks (i.e. PRE, POST and training blocks). Additionally in PRE and POST training (see below), participants were asked to evaluate their confidence on a scale of 1 to 5 
after they provided their main response. No feedback was provided. Inter-trials intervals (ITI) ranged from 0.6 to $0.8 \mathrm{~s}$. Crucially, during PRE and POST training, all participants (V, AV and AVn) were tested with visual stimuli i.e. without any sound. In the PRE training block, the initial coherence discrimination threshold of participants was assessed by testing seven levels of visual RDK coherence, namely: $15 \%, 25 \%, 35 \%, 45 \%, 55 \%, 75 \%$ and $95 \%$.

PRE training was followed by four training blocks of 5 min each for a total of 20 minutes of training. The training consisted of the same task as in the PRE and POST training. Stimuli were presented without any sound (V group), with correlated acoustic textures (AV group) or with uncorrelated acoustic noise (AVn group). During training, four visual RDK coherence levels were tested and corresponded to $\pm 10 \%$ and $\pm 20 \%$ of the individual's PRE training coherence discrimination threshold. Hence, participants underwent individualized training. 28 trials of each coherence level were presented for a total of 112 trials in a given training block. The training was followed by a 12 minutes post-training session (POST training). POST training evaluated participants' threshold after training. As in the PRE training block, POST training was solely visual for all groups (V, AV, and AVn) and participants were asked to rate their confidence after providing their response. In PRE and POST training, a total of 196 trials were tested (28 trials / coherence level).

Participants never received any feedback after the familiarization trials. Rest periods were provided to participants after each experimental block. Crucially, the color of the most coherent RDK was counterbalanced and the directions of coherent motion were pseudorandomized across all trials - hence, the direction of motion was orthogonal to the main task ("coherence").

After the POST training block, a localizer was used to provide an accurate source localization of the hMT+ area with MEG. For this, participants passively viewed a sequence in which a RDK was either fully incoherent for $1.5 \mathrm{~s}$ or fully incoherent for $0.5 \mathrm{~s}$ followed by $95 \%$ 
coherence for 1s. Each sequence was presented 60 times for a total of 120 presentations during the localizer block.

\subsection{Visual stimuli}

The red and green RDKs were individually calibrated to isoluminance using HFP. To prevent local tracking of dots, a white fixation cross was located at the center of a $4^{\circ}$ gray disk acting as a mask (Figure 1B). RDKs were presented within an annulus of $4^{\circ}-15^{\circ}$ of visual angle. Dots had a radius of $0.2^{\circ}$. The flow of RDKs was 16.7 dots per $\mathrm{deg}^{2} \times \mathrm{sec}$ with a speed of $10 \%$ s. During the first 0.3 to $0.6 \mathrm{~s}$ of a given trial, both RDKs were incoherent $(0 \%$ of coherent motion). The duration of the incoherent phase was pseudo-randomized across each trial in order to increase the difficulty of the task specifically by preventing participants from expecting the onset of the transition from incoherent to coherent motion. After the incoherent phase, one RDK became more coherent than the other during one second. The direction of coherent dots was comprised within an angle of $45^{\circ}-90^{\circ}$ around the azimuth. $50 \%$ of the trials were upward coherent motion and the remaining $50 \%$ of the trials were downward coherent motion. At each frame, $5 \%$ of all dots were randomly reassigned to new positions and incoherent dots to a new direction of motion. Dots going into collision in the next frame were also reassigned a new direction of motion. On average, the life-time of the dots was set to 180 ms ( \pm 165 s.d.) and could be approximated by a Weibull distribution of parameters $\alpha=172 \mathrm{~ms}$ and $\beta=1.04$.

\subsection{Auditory stimuli}


A sample spectrogram of an auditory stimulus is provided in Figure 1C. All auditory stimuli were created with a sampling frequency of $44.1 \mathrm{kHz}$. Acoustic textures (Overath et al., 2010) were developed to be analogous and congruent to visual RDKs. Specifically, each visual dot was designed as if to emit a sound $s(t)$ corresponding to a linear frequency-modulated ramp whose slope depended on the direction taken by the visual dot: $s(t)=\cos \left(2 \pi \cdot \mathrm{e}^{\text {slope.t+log }\left(f_{0}\right)} \cdot t\right)$ where slope $=2 \tan (\varphi)$. The angle between the direction of the dot and the azimuth is denoted by $\varphi$ and the initial sound frequency is denoted by $f_{0}$. For instance, a visual motion direction of $45^{\circ}$ would correspond to a slope of 2 octaves per second in the acoustic space. The maximal slope authorized in acoustic space was set to 16 octaves/s corresponding visual motion directions of $82.9^{\circ}-90^{\circ}$. Each $\operatorname{ramp} f_{0}$ was attributed according to the initial vertical position of the corresponding visual dot: the lower the position of the dot on the screen, the lower the $f_{0}$ in acoustic space. Hence, a visual dot moving upwards was associated with an ascending acoustic ramp, whereas a visual dot moving downwards was associated with a descending acoustic ramp. Note that sensory substitution devices (e.g., the vOICe (Meijer, 1992) and the EyeMusic (Levy-Tzedek et al., 2012a)) have also started capitalizing on such intuitive perceptual associations (Melara and O’Brien, 1987; Maeda et al., 2004)). The auditory frequencies that were used ranged between 200 and $5000 \mathrm{~Hz}$. Should a ramp cross one of these limits, it "continued" at the other extreme of this frequency band. The duration of a ramp was identical to the life-time of a visual dot. Importantly, when visual dots moved coherently, they did not necessarily emit the same sounds because the initial auditory frequencies were likely different. However, the variations of the acoustic elements (i.e. the slopes of the ramps) were identical. Hence, the quantification of visual coherence in RDK matched the proportion of ramps having the same slope in acoustic space.

In the AVn training, acoustic noises with the same duration and amplitude of acoustic textures were used to test whether the simple presentation of a sound could account for the results. We 
specifically designed this control to test the hypothesis that the introduced correspondences (matching the spectral characteristics of the acoustic textures with the spatial characteristics of the visual stimuli) were relevant for learning in this task. Hence, the noise $y(t)$ was conservatively designed within the same frequency range $(200-5000 \mathrm{~Hz})$ as the acoustic textures used in the AV group: $\mathrm{y}(t)=\cos \left(2 \pi \cdot \mathrm{e}^{\operatorname{rand} *\left(\log \left(f_{\max }\right)-\log \left(f_{\min }\right)\right)+\log \left(f_{\min }\right)} \cdot t\right)$, where rand denotes the uniformly distributed pseudorandom function whose values are contained in the interval $[0,1], f_{\min }=200 \mathrm{~Hz}$ and $f_{\max }=5000 \mathrm{HZ}$.

\subsection{Psychophysical analysis}

The coherence discrimination threshold was set to $75 \%$ of correct performance and quantified by fitting a Weibull function (Wichmann and Hill, 2001) to each individual's data using: $\psi(\operatorname{coh}, \alpha, \beta)=1-0.5 e^{-\left(\frac{c o h}{\alpha}\right)^{\beta}}$. With $\operatorname{coh}$ as motion coherence level, $\psi$ as the fitted psychometric function, and $\alpha$ and $\beta$ the parameters determined by the damped Gauss-Newton method. A mixed-design ANOVA containing the within-subject factor test (pre-and posttraining) and the between-subjects factor training group (V, AV and AVn) was carried out separately on the perceptual thresholds, the confidence ratings and the RTs using the software $R$ (R Core Team 2013). If a main effect of the factor test was found, a post-hoc analysis using Bonferroni-corrected paired t-tests on each group was conducted. Likewise, a main interaction between the two factors test and training was analyzed with a Bonferroni-corrected twosampled t-test between each pair of groups.

\subsection{MEG data acquisition}

Brain magnetic fields were recorded in a MSR using a 306 MEG system (Neuromag Elekta LTD, Helsinki). MEG recordings were sampled at $2 \mathrm{kHz}$ and band-pass filtered between $0.03-$ $600 \mathrm{~Hz}$. Four head position coils (HPI) measured the head position of participants before each block; three fiducial markers (nasion and pre-auricular points) were used for digitization and 
anatomical MRI (aMRI) immediately following MEG acquisition. Electrooculograms (EOG, horizontal and vertical eye movements) and electrocardiogram (ECG) were simultaneously recorded. Prior to the session, 5 minutes of empty room recordings were acquired for the computation of the noise covariance matrix.

\subsection{Anatomical MRI acquisition and segmentation}

The T1 weighted aMRI was recorded using a 3-T Siemens Trio MRI scanner. Parameters of the sequence were: voxel size: $1.0 \times 1.0 \times 1.1 \mathrm{~mm}$; acquisition time: $466 \mathrm{~s}$; repetition time TR $=2300 \mathrm{~ms}$; and echo time $\mathrm{TE}=2.98 \mathrm{~ms}$. Cortical reconstruction and volumetric segmentation of participants' T1 weighted aMRI was performed with FreeSurfer (http://surfer.nmr.mgh.harvard.edu/). This includes: motion correction, average of multiple volumetric T1 weighted images, removal of non-brain tissue, automated Talairach transformation, intensity normalization, tessellation of the gray matter white matter boundary, automated topology correction, and surface deformation following intensity gradients (Dale et al., 1999; Fischl and Dale, 2000). Once cortical models were complete, deformable procedures could be performed including surface inflation (Fischl et al., 1999a) and registration to a spherical atlas (Fischl et al., 1999b). These procedures were used with MNE (Gramfort et al., 2013a, 2013b) to morph current source estimates of each individual onto the FreeSurfer average brain for group analysis.

\subsection{MEG data preprocessing and Event Related Fields (ERFs) analysis}

Data analysis was done in accordance with accepted guidelines for MEG research (Gross et al., 2012). Signal Space Separation (SSS) was carried out using MaxFilter to remove external interferences and noisy sensors (Taulu and Simola, 2006). Ocular and cardiac artifacts were 
removed by creating signal space projections (SSP) based on average-locked responses to the QRS heart complex recorded with ECG and to the blinks recorded with EOG. About 2 to 3 components were projected out of the raw data. Next, raw data were band-pass filtered between 1-40 Hz and down-sampled to $250 \mathrm{~Hz}$.

For the main analysis, data were epoched from $-200 \mathrm{~ms}$ (baseline) to $+1000 \mathrm{~ms}$ around the onset of coherent RDK. Epochs were averaged for each individual according to the conditions of interest, namely: across all coherence levels (196 trials) or for each coherence level (28 trials). Trials corrupted by muscle or movement artifacts (less than $10 \%$ of all trials) were rejected by visual inspection using Fieldtrip (Oostenveld et al., 2011). Additionally, epochs were averaged according to each individual's pre- and post-training thresholds into three categories: 'hard' (coherence levels below the POST-training threshold), 'learned' (coherence levels between the PRE- and the POST-training thresholds) and 'easy' (coherence levels above the PRE-training threshold). Evoked responses were smoothed with a Savitzky-Golay filter (Savitzky and Golay, 1964) consisting of fitting a $2^{\text {nd }}$ order polynomial to each sliding window of 35 samples. This procedure is approximately equivalent to the application of a low-pass filter of $3 \mathrm{~dB}$ cutoff frequency set to $37.5 \mathrm{~Hz}$ (Schafer, 2011) without reduction of peak amplitudes.

\subsection{MRI-MEG coregistration and source reconstruction}

The co-registration of MEG data with an individual's aMRI was carried out by realigning the digitized fiducial points with the multimodal markers visible in MRI slices. We used a twosteps procedure to insure reliable coregistration between MRI and MEG coordinates: using MRILAB (Neuromag-Elekta LTD, Helsinki), fiducials were aligned manually with the multimodal markers visible on the MRI slice; an iterative procedure was then used to realign 
all digitized points (about 30 more supplementary points distributed on the scalp of the subject were digitized) with the scalp tessellation using the mne_analyze tools within MNE (Gramfort et al., 2013b). Individual forward solutions for all source reconstructions located on the cortical sheet were next computed using a 3-layers boundary element model (Hämäläinen and Sarvas, 1989; Mosher et al., 1999) constrained by the individual aMRI. Cortical surfaces were extracted with FreeSurfer and decimated to about 5,120 vertices per hemisphere with 4.9 mm spacing. The forward solution, noise and source covariance matrices were used to calculate the depth-weighted (parameter $\gamma=0.8$ ) and noise-normalized dynamic statistical parametric mapping (dSPM) (Dale et al., 2000) inverse operator. This unitless inverse operator was applied using a loose orientation constraint on individuals' brain data (Lin et al., 2006) by setting the transverse component of the source covariance matrix to 0.4 . The reconstructed current orientations were pooled by taking the norm, resulting in manipulating only positive values. The reconstructed dSPM estimates time series were interpolated onto the FreeSurfer average brain for group analysis (Fischl et al., 1999b) and common referencing. 
A
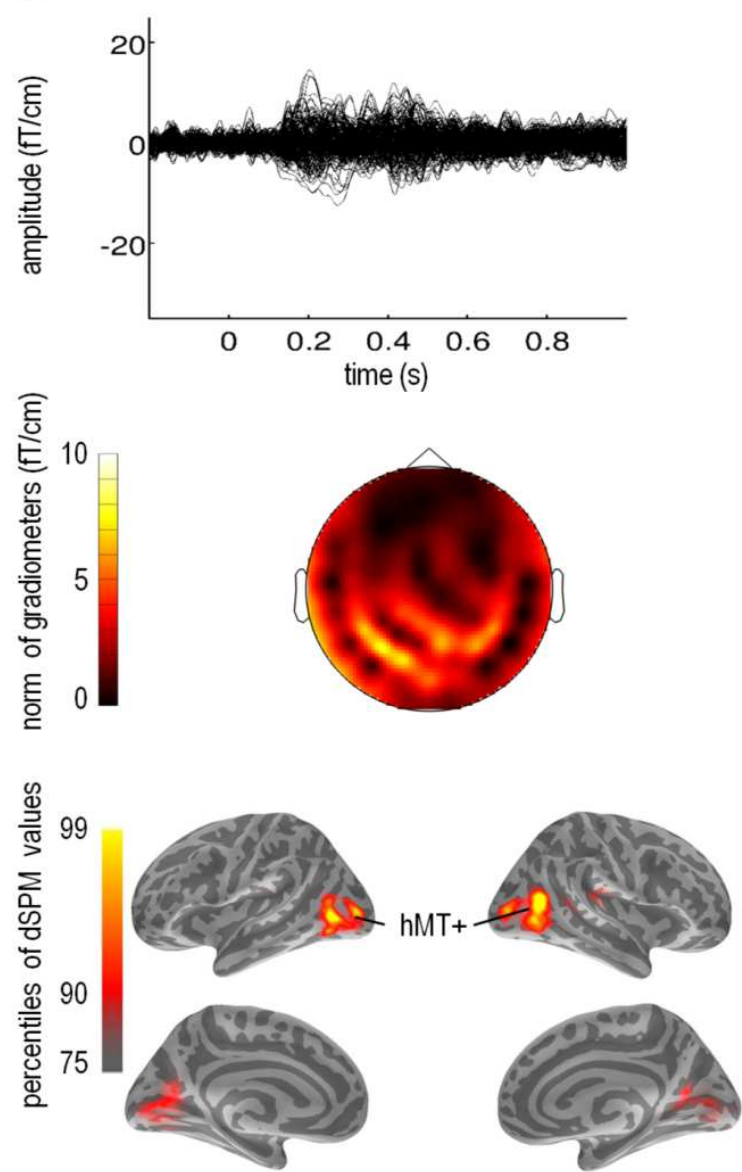

B
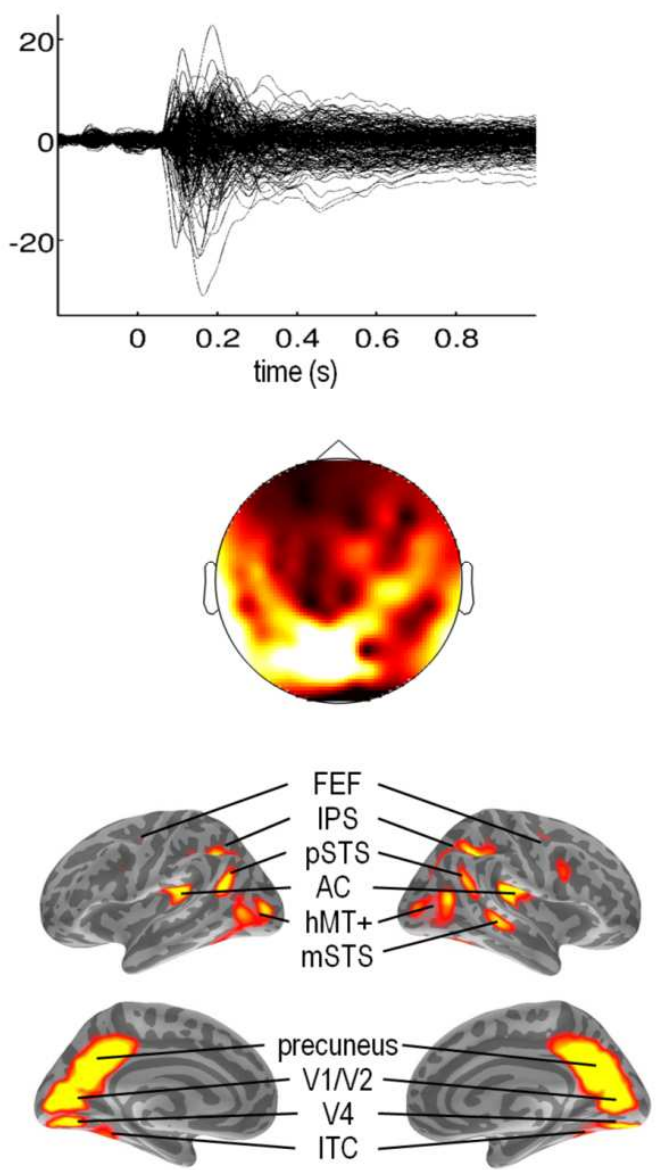

Figure 2. MNE (dSPM) source reconstruction and regions of interest (ROIS). Panel A: Evoked Response Fields (ERF) in sensor space (gradiometers) obtained in response to the presentation of the hMT+ localizer. Specifically, we report the evoked component obtained by subtracting the ERF in response to fully incoherent motion (0\%) from the ERF in response to a 95\% coherent motion. These data were collected during the localizer block and pooled across all individuals (i.e. all three training groups: $\mathrm{V}, \mathrm{AV}$, and Avn; $\mathrm{n}=36$ participants).The time course of all gradiometers is provided in the top graph. The topography of the differential evoked component averaged over 100 to $300 \mathrm{~ms}$ post-stimulus onset is provided for the norm of the gradiometers in the middle graph. The corresponding current source estimates (MNE, dSPM) is provided in the bottom graph. Panel B: ERF in sensor space (gradiometers) obtained in response to the presentation of incoherent visual RDKs. PRE and POST training data were pooled together across all three training groups $(n=36)$ in order to define the regions of interest (ROIs). The time course of the evoked response obtained at the onset of all visual stimuli is depicted in the top graph for all gradiometers. Distinct evoked components can be seen starting at $100 \mathrm{~ms}$. The topography of the ERF is provided in the middle graph for the norm of gradiometers averaged over 100 to 300 ms post-incoherence onset. The corresponding current source estimates (MNE, dSPM) are provided in the bottom graph. The extent of a given label or region of interest (ROI) in source space was defined by thresholding the dSPM estimates at the 90th percentile of all dSPM values. FEF: Frontal Eye Field. IPS: Inferior Parietal Sulcus. pSTS: posterior Superior Temporal Sulcus. AC: Auditory Cortex. mSTS: middle Superior Temporal Sulcus. ITC: Inferior Temporal Cortex. 


\subsection{Functional localizer for hMT+ and selection criteria for the regions of interest (ROIs)}

One major prediction in this study was the that perceptual improvements in coherence discrimination thresholds would be commensurate with post-training activity in hMT+ which is known to be responsive to global (Watson et al., 1993; Zeki et al., 1993; Tootell et al., 1995) and translational (Morrone et al., 2000) motion processing. Hence, after source reconstruction, hMT+ was localized on a per individual basis by contrasting the current source estimate obtained to the presentation of $95 \%$ coherent motion against the incoherent $(0 \%)$ portion of the hMT+ localizer. Specifically, the evoked response fields (ERFs) elicited by the transition to full coherence in the visual display (i.e. going from $0 \%$ to $95 \%$ coherence) were contrasted with the ERFs elicited at the same latency but in the absence of transition (i.e. $0 \%$ of coherence). A first inspection of the ERF contrast averaged over all individuals in sensor space (Fig. 2A, upper and middle panel) showed a main evoked response spanning $\sim 100$ to $\sim 300 \mathrm{~ms}$ post-transition onset. The evoked response was source reconstructed using MNE-dSPM; the extent of the area hMT+ in source space was determined by thresholding the average source estimate amplitudes over $100-300 \mathrm{~ms}$ above the $90^{\text {th }}$ percentile of all $\mathrm{dSPM}$ values covering the entire cortex (Fig. 2A, lower panel).

Figure $2 \mathrm{~B}$ reports additional regions of interest (ROI) or labels which were identified at the group-level by source reconstructing the grand average evoked field response to the presentation of incoherent visual RDKs which combined data from all three training groups $(\mathrm{V}, \mathrm{AV}$ and $\mathrm{AVn})$ in the pre- and in the post-training sessions. The most responsive areas (selected by thresholding to the 90th percentile of all dSPM values) were manually labeled using the Freesurfer neuroanatomical parcellation. The obtained ROIs comprised: bilateral primary and secondary visual cortices (V1 and V2, respectively), precuneus, visual area V4, 
hMT+, Inferior Temporal Cortex (ITC), Auditory Cortex (AC), posterior Superior Temporal Sulcus (pSTS), Inferior Parietal Sulcus (IPS), frontal eye-field (FEF) and the right middle Superior Temporal Sulcus (mSTS). The time courses reported in a label were computed by averaging dSPM estimate time courses over all vertices within the label. It is worth noting that dSPM values are here only positive and hence do not cancel out after averaging.

\subsection{Neurometric functions}

For each individual, neurometric curves (Britten et al., 1992; Gold et al., 2010) were computed using pre- and post-training current source estimates in hMT+ averaged between 200 and $500 \mathrm{~ms}$ after coherence onset. The amplitudes of the cortical responses to the presentation of each of the 7 levels of coherence were fitted by a Weibull function: $Y(\operatorname{coh}, M, m, \alpha, \beta)=M-(M-m) e^{-\left(\frac{c o h}{\alpha}\right)^{\beta}}$. With $\operatorname{coh}$ as motion coherence level, $Y$ as the fitted neurometric function, and $M, m, \alpha$ and $\beta$ the parameters determined by the damped Gauss-Newton method. Individual neurometric thresholds were defined as the level of coherence corresponding to the half-amplitude of the sigmoid.

\subsection{Statistics}

The effect of training was tested using the POST minus PRE contrasts across all coherence levels separately for each ROI using F-tests combined with non-parametric permutation tests (Maris and Oostenveld, 2007) that provide corrected p-values for multiple comparisons. For each signed permutation $(\mathrm{N}=20000)$, time clusters were defined on the basis of temporal adjacency by regrouping samples whose F-statistic was larger than 3.3 (i.e. p-value inferior to 0.05 for an F-test with $2 \times 33$ degrees of freedom). Cluster-level statistics were then calculated 
by taking the sum of the F-values within the cluster. Only temporal clusters with corrected pvalues $\leq 0.05$ are reported. The significance of the contrasts were also tested in each group using non-parametric pairwise two-tailed permutation tests with the cluster threshold set to 2.2 (i.e. p-value inferior to 0.05 for a two-sided t-test with 11 degrees of freedom).

All correlation tests were assessed with Pearson correlation coefficients $\rho$ under the null hypothesis $H_{o}: \rho=0$ and with the alternative $H_{1}: \rho \neq 0$ using a Student t-test on the statistic $t=\rho \sqrt{n-2} / \sqrt{1-\rho^{2}}$, where $n$ is the number of samples. Outliers were automatically detected and rejected by using a leave-one-out approach (Weisberg, 2005) consisting of estimating the distribution $N(m, \sigma)$ of residuals based on (n-1) observations (each observation is left out one after another). Extreme residuals (i.e. above and below $m \pm k . \sigma$, where $k=2.5$ is considered to be a reasonable choice (Rousseeuw and Leroy, 1987)) are identified and the corresponding observations set as outliers. 



Figure 3. Behavioral results as a function of training type (V, AV, or AVn). Panel A: Mean psychometric curves ( \pm 1 s.e.m.) before (PRE, light grey) and after (POST, dark grey) training. Mean performance as a function of visual coherence levels in V (top), AV (middle) and AVn (bottom) training. Each group included 12 individuals. The mean perceptual threshold corresponds to the mean coherence value of one RDK population with a correct response rate of $75 \%$ (black dashed line). Perceptual threshold improvements were significant in all groups (black arrows). Panel B: Each individual psychometric curve was fitted with a Weibull function in order to extract an individual's discrimination threshold. Here, we report the mean threshold obtained in PRE (light grey) and POST (dark gray) training data. In PRE training, no significant differences in perceptual thresholds were found among the three groups $\left(\mathrm{F}_{2,33}=1.12, \mathrm{p}=0.34\right.$, mixed-design ANOVA); in POST training, all three groups showed a significant difference in their mean perceptual threshold as compared to the PRE training data $\left(\mathrm{F}_{1,33}=132, \mathrm{p}=4.5 \mathrm{e}-13\right.$, mixed-design ANOVA). A significant interaction between training groups was found $\left(\mathrm{F}_{2,33}=8.3, \mathrm{p}=1.2 \mathrm{e}-3\right.$, mixed-design ANOVA): as can readily be seen, the post-training threshold in the AV group was significantly lower than the one obtained in the V and in the AVn groups (post-hoc analysis, with Bonferroni correction). Panel C: Mean confidence ratings across all coherence levels in the V, AV and AVn groups in PRE and POST training. In PRE, no significant differences of confidence ratings were observed across the three groups $\left(\mathrm{F}_{2,33}=0.61, \mathrm{p}=0.55\right.$, mixed-design ANOVA). In POST, a significant improvement of confidence rating was observed $\left(\mathrm{F}_{1,33}=7.2, \mathrm{p}=0.011\right)$ but solely in the $\mathrm{V}$ group after post-hoc analysis (Bonferroni). No significant interaction was found between training groups $\left(\mathrm{F}_{2,33}=1.35, \mathrm{p}=0.27\right)$. Panel $\mathrm{D}$ : Mean reaction times (RTs) across all coherence levels in the V, AV and AVn groups in PRE and POST training. In PRE, no significant differences of RTs was observed across training groups $\left(F_{2,33}=0.007, p=0.99\right.$, mixeddesign ANOVA). In POST training, all three training groups showed a significant decrease of their RT $\left(\mathrm{F}_{1,33}=\right.$ $95, \mathrm{p}=3 \mathrm{e}-11)$ but no significant interaction was found between the training groups $\left(\mathrm{F}_{2,33}=1.5, \mathrm{p}=0.23\right)$. '*': 
corrected $\mathrm{p}$ value inferior to 0.05 ; ‘**': corrected $\mathrm{p}$ value inferior to 0.01 ; '***': corrected $\mathrm{p}$ value inferior to 0.001 .

\section{RESULTS}

\subsection{AV training improves performance best}

First, we tested whether participants improved on the task by comparing each individual's coherence discrimination threshold before and after training (Fig. 3A) using a mixed-design ANOVA (see Materials and Methods). The post-training thresholds of individuals are also given as a function of pre-training thresholds in all three groups in Supp. Fig. 1A. In pretraining, all participants performed similarly well on the coherence discrimination task and the observed perceptual thresholds did not significantly differ between the three groups of individuals $(F(2,33=1.12), p=0.34)$. In all three groups, training successfully improved the performance of participants when comparing pre- and post-training coherence discrimination threshold $(F(1,33)=132, p=4.5 \mathrm{e}-13)$. Crucially, a significant interaction between training type and pre/post-training $(F(2,33)=8.3, p=1.2 \mathrm{e}-3)$ showed that perceptual improvements in the $\mathrm{AV}$ group significantly outperformed those observed in the $\mathrm{V}$ and $\mathrm{AVn}$ groups only in post-training (Fig. 3B, post-hoc analysis with Bonferroni correction). It is important to note that since the pre- and post-training coherence discrimination thresholds were solely established on the basis of visual RDK presentation, any differences in post-training performance can be solely accounted for by training history and not by the mere presence of $\mathrm{AV}$ stimuli. Additionally, no significant changes in the slope of the psychometric function (i.e. the beta parameter) were observed after training $\left(\mathrm{F}_{1,33}=0.36, \mathrm{p}=0.55\right.$, mixed-design ANOVA), no interactions between the slope of the psychometric function and the training type was observed $\left(F_{2,33}=1,63, p=0.21\right)$ (cf. Supp. Fig. 1B) and no significant correlation between the differences in slope and perceptual threshold was observed in either group (as 
reported in Supp Fig 1C). Altogether, these results strongly support a genuine change in participants' sensitivity to motion coherence.

A similar mixed-design ANOVA was carried out using the reaction times (RTs) of individuals as dependent variable. All participants showed shorter post-training RTs (Fig. 3B, $\mathrm{F}(1,33)=132, \mathrm{p}=4.5 \mathrm{e}-13)$ but no significant interactions between $\mathrm{RT}$ and training type was found $(\mathrm{F}(2,33)=1.5, \mathrm{p}=0.23)$. The mean RT for each group was otherwise comparable before and after training $(\mathrm{F}(2,33)=0.007, \mathrm{p}=0.99$, mixed-design ANOVA $)$.

During pre- and post-training, participants were asked to rate their confidence level following their coherence discrimination response. Contrasting the mean confidence ratings before and after training across all coherence levels (Fig. 3D), a mixed-design ANOVA revealed a main effect of session (2: pre- and post-training) on confidence rating $(F(1,33)=7.2, p=0.011)$; no significant interaction between session and training type was observed $(F(2,33)=1.35, p=$ 0.27). However, post-hoc analysis revealed that the main effect of session could be solely attributed to the $\mathrm{V}$ group although the groups were not statistically distinguishable $(\mathrm{F}(2,33)=$ $0.61, p=0.55)$. Thus, and surprisingly, while AV learners showed the largest improvement in performance after training, they were not aware of their own improvements contrarily to participants in the V group (Figure 3D). 

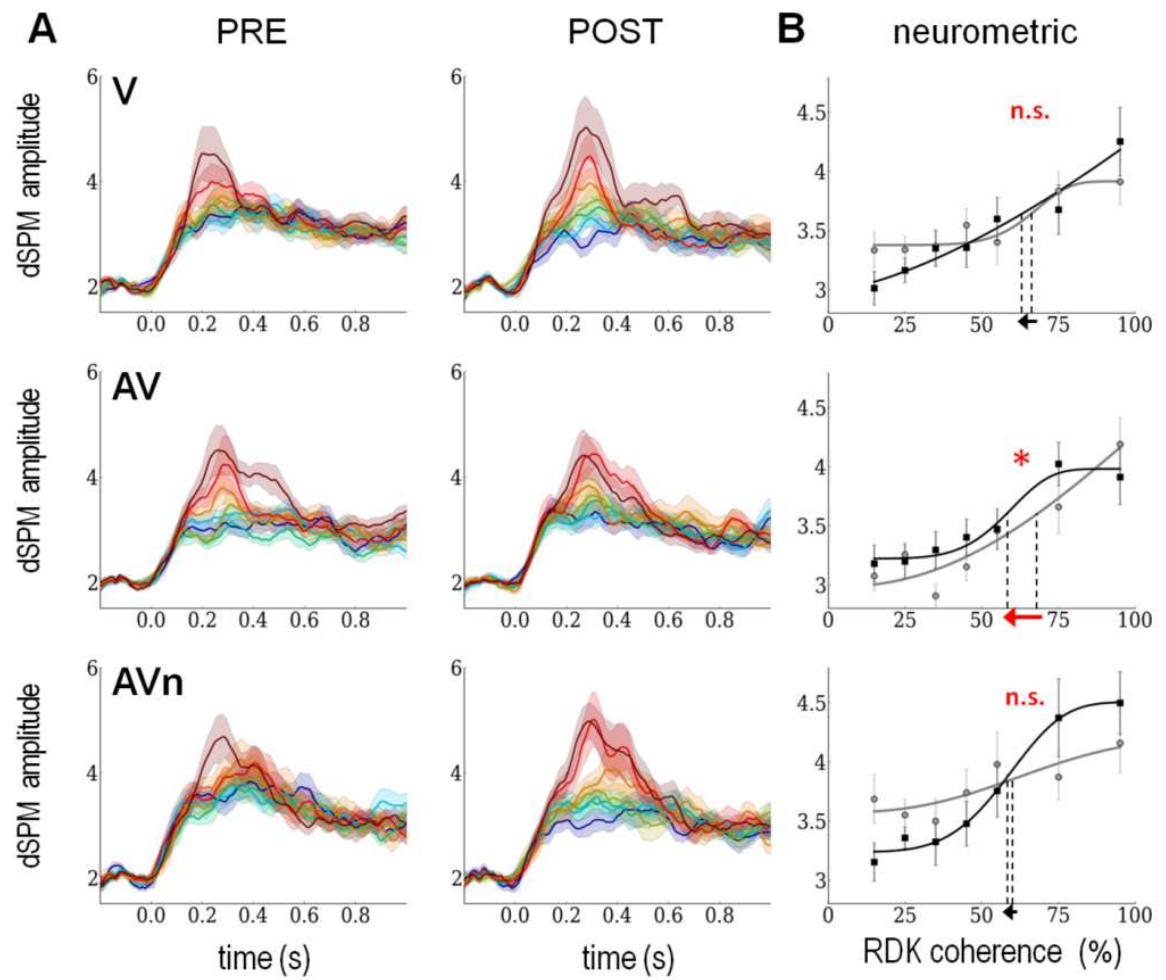

RDK coherence (\%): $\begin{array}{lllllll}15 & 25 & 35 & 45 & 55 & 75 & 95\end{array}$

PRE POST

\section{Figure 4. Pre- and post-training hMT+ response as a function of visual RDK coherence levels and}

neurometric functions. Panel A: Time course of current source estimates (dSPM amplitudes) in bilateral hMT+ for the different training groups (V: top, AV: middle and AVn: bottom panels) as a function of RDK coherence levels (cf. color scheme legend). Data obtained in the pre- and post-training blocks are reported in the left and right panels, respectively. A prominent evoked response peaking at $\sim 200 \mathrm{~ms}$ post-coherence onset can readily be seen in all groups and for all coherence levels. Additionally, the higher the visual coherence, the higher the amplitude of the cortical response. While the profile of responses was similar across the three groups before training, a distinct response pattern was found after training. Specifically, the V and AVn showed an increased spread of the response amplitudes as a function of visual coherence levels whereas the AV group did not. In order to characterize this response pattern, a 200 to $500 \mathrm{~ms}$ time period was selected, averaged and used to construct the individual neurometric curves consisting of the amplitude of the current source estimate as a function of stimulus coherence level. Panel B: The current source estimates (dSPM) in hMT+ were averaged from 200 to $500 \mathrm{~ms}$ post-coherence onset as a function of the seven coherence levels in $\mathrm{V}$ (top), AV (middle) and AVn (bottom). This quantification was performed separately for the pre- and post-training data (grey and black, respectively). The brain response in hMT+ of each individual was quantified for each coherence levels. To obtain the neurometric function of an individual, the amplitudes of the current source estimates in hMT+ were plotted as a function of visual RDK coherence level. Each neurometric function allowed deriving a neurometric threshold via Weibull fits (i.e. the level of coherence corresponding to half the amplitude of the sigmoid curve). For illustration purposes, we report the averaged fits together with the grand average data. The neurometric thresholds obtained in pre- and post-training were compared by carrying out a two-tailed paired t-test. Using this method, we show that neither $\mathrm{V}$ or $\mathrm{AVn}$ showed a significant change in neurometric threshold $\left(\mathrm{V}: \mathrm{t}_{11}=-0.2, \mathrm{p}=\right.$ 
0.84; AVn: $\left.\mathrm{t}_{11}=-0.36, \mathrm{p}=0.72\right)$ whereas AV showed a significant decrease of neurometric threshold $\left(\mathrm{t}_{11}=-2.34\right.$, $\mathrm{p}=0.039$ ). This suggests that the neural response to a given coherence level, hence the neural selectivity in $\mathrm{hMT}+$, has significantly changed according to the type of training provided to the participants. Specifically, the sensitivity to coherence discrimination in hMT+ significantly improved solely in the AV group.

\section{2 hMT + plasticity and sensitivity profiles}

According to previous reports (Ahlfors et al., 1999; Lam et al., 2000; Maruyama et al., 2002; Nakamura, 2003; Aspell et al., 2005; Amano et al., 2006; Händel et al., 2007; Mercier et al., 2009), the amplitude of the evoked responses in hMT+ increases with RDK coherence levels irrespective of participants' performance. As a first approach, we thus separately classified trials as a function of the physical coherence of the visual stimuli (i.e. 7 RDK coherence levels ranging from $15 \%$ to $95 \%$ ) per training type and as a function of pre- and post-training. After source reconstruction, a similar pattern of response in hMT+ could be seen in all three groups with a clear evoked response in hMT+ from $\sim 200 \mathrm{~ms}$ to $\sim 500 \mathrm{~ms}$ post-stimulus onset (Fig. 4A).

One hypothesis on the origin of perceptual improvements was that the sensitivity of hMT+ response to RDK coherence would improve after training. When contrasting the average $\mathrm{hMT}+$ response profiles in pre- and post-training (Fig. 4A), the spread of the hMT+ response amplitudes as a function of RDK coherence after training appeared much larger in the $\mathrm{V}$ and AVn groups; surprisingly however, the AV group did not show such changes. A linear regression of the hMT+ amplitude estimates as a function of RDK coherence levels clearly indicated that the AV group showed no significant differences in pre- $v s$. post-training (Supp. Fig S2A, beta values). At first glance then, these results suggested that the perceptual improvements in the AV group could not be accounted for by hMT+ plasticity.

Using a similar approach to psychometric characterization, it is well known that hMT+ sensitivity to coherent motion can be characterized by a neurometric function (Britten et al., 
1992); one advantage of neurometric thresholds is that they are comparable to psychometric functions depending on the experimental conditions (Britten et al., 1992). Hence, to better understand the evolution of the response profiles observed in hMT+, we selected the 200-500 ms time period post-coherence onset and fitted a Weibull function to the averaged source estimate amplitudes as a function of RDK coherence levels on a per individual basis, and separately for pre- and post-training data (see Materials and Methods). From each fit, the neurometric threshold of an individual could thus be defined as the stimulus coherence level corresponding to half the amplitude of the sigmoid curve (see Supp. Fig S2B for examples of individual fits and Fig. 4B for the group data). Using this procedure, a significant decrease in neurometric threshold was observed solely in the AV group $\left(t_{11}=-2.34, p=0.039\right.$; Fig. 4B).

Altogether, these results suggest a particular neural strategy in hMT+ that depends on the training history of participants, namely: in V and AVn, sensitivity affected the extreme levels of RDK coherence whereas in AV a better selectivity was seen for RDK coherence close to perceptual threshold. Although no direct correlation could be found between neurometric and psychometric thresholds when separately considering the pre- and post-training data, significant correlations between perceptual and neurometric threshold changes were observed in each group and across all individuals irrespective of their training history (Fig. 5). 


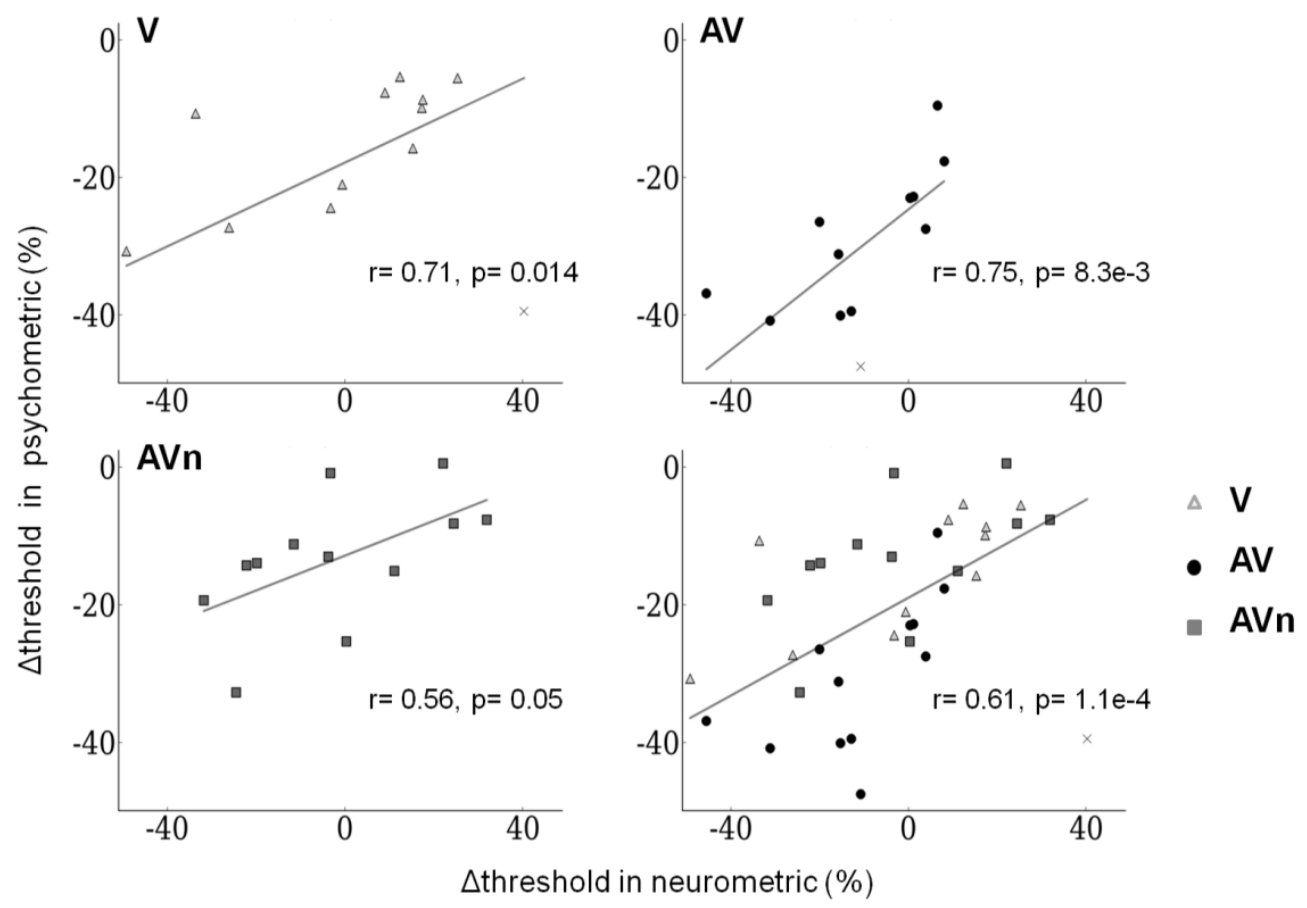

Figure 5. Changes in visual coherence discrimination thresholds as a function of changes in neurometric thresholds pre- and post-training. Differences in the perceptual thresholds of individuals before and after training are reported as a function of the changes in neurometric thresholds on a per training group basis (V: top left; AV: top right; AVn: bottom left; all groups combined: bottom right). In all three training groups, the individual improvements in coherence discrimination thresholds were significantly correlated with the observed changes in neurometric thresholds derived from source estimate activity in bilateral hMT+. Specifically, correlations were the highest in the $\mathrm{V}$ and $\mathrm{AV}$ groups ( $\mathrm{V}: \mathrm{r}=0.71, \mathrm{p}=0.014$ and $\mathrm{AV}: \mathrm{r}=0.75, \mathrm{p}=8.3 \mathrm{e}-3$ ) but also in the $\mathrm{AVn}$ group $(\mathrm{r}=0.56, \mathrm{p}=0.05)$. When grouping all individuals, a significant correlation was preserved (bottom right, $r=0.61, p=1.1 e-4$ ) . ' $x$ ' denotes statistical outliers.

Altogether, these results strongly suggest that the hMT+ response profile to a given RDK coherence level significantly changed as a function of the training history of the individual. Crucially, the sensitivity of hMT+ response to RDK selectively improved in the AV group but not in the $\mathrm{V}$ and control AVn groups.

\subsection{Enhanced hMT+ selectivity for learned coherence levels only after AV training}


In order to narrow down the specific effects of training on the hMT+ response profile, we classified data according to the perceptual improvement of each individual. Specifically, participants underwent individualized training so that each individual was trained on a selected set of four RDK coherence levels based on the initial discrimination threshold measured in pre-training. Hence, participants were not trained on the same set of coherence levels although all were tested on the same 7 coherence levels in pre- and post-training blocks. On this basis, we classified the 7 RDK coherence levels into three sets solely based on their learned discriminability - i.e. irrespective of the physical RDK coherence levels - in order to sort data in the pre- and post-training blocks. The three categories were 'hard', 'easy' and 'learned': the 'hard' category consisted of all stimuli that remained below an individual's perceptual threshold after training - i.e. RDK coherence levels that never benefitted from training and did not become perceptually discriminable for a given participant. Conversely, the 'easy' category corresponded to those stimuli that were already above the discrimination threshold before training. Most importantly, the 'learned' category consisted of all RDK coherence levels that became discriminable - i.e. above the discrimination threshold of the individual after training. We then hypothesized that plasticity should be precisely reflected by a change of neural activity elicited by the 'learned' category and not others.

Examining the changes of hMT+ responses over 200 to $500 \mathrm{~ms}$ revealed significant differences between the three groups in the 'learned' $\left(\mathrm{F}_{2,33}=5.4, \mathrm{p}=0.0091\right)$ and in the 'hard' $\left(\mathrm{F}_{2,33}=4.8, \mathrm{p}=0.015\right)$ categories. Specifically, the $\mathrm{V}$ and $\mathrm{AVn}$ groups shared a similar pattern of responses across all three categories: opposite variations in 'hard' and 'easy' categories were observed in the $\mathrm{V}$ and $\mathrm{AVn}$ groups consistent with the increased spread of hMT+ response as a function of RDK coherence levels (Fig. 4A). In contrast, and consistent with the reported shifts in neurometric thresholds (Fig. 4B), the AV group presented a significant change in response profile to the 'learned' category $\left(\mathrm{t}_{11}=3.23, \mathrm{p}_{\mathrm{cor}}=2.4 \mathrm{e}-2\right.$, bilateral paired $\mathrm{t}$-test with 
Bonferroni correction). In addition, these results were confirmed by a finer analysis of the entire time course differences in hMT+ (Fig. 6, first column) when carrying out a pairwise cluster permutation algorithm (cf. Table 1): AV was indeed the only group to show a significant response increase for the 'learned' coherence levels from 160 to 390 ms postcoherence onset.

With this analysis, we thus consistently observe that only AV trained individuals showed a significant change in hMT+ activity that directly related to the observed perceptual improvements and to those stimuli that underwent a significant change in perceptual discriminability.


learned easy
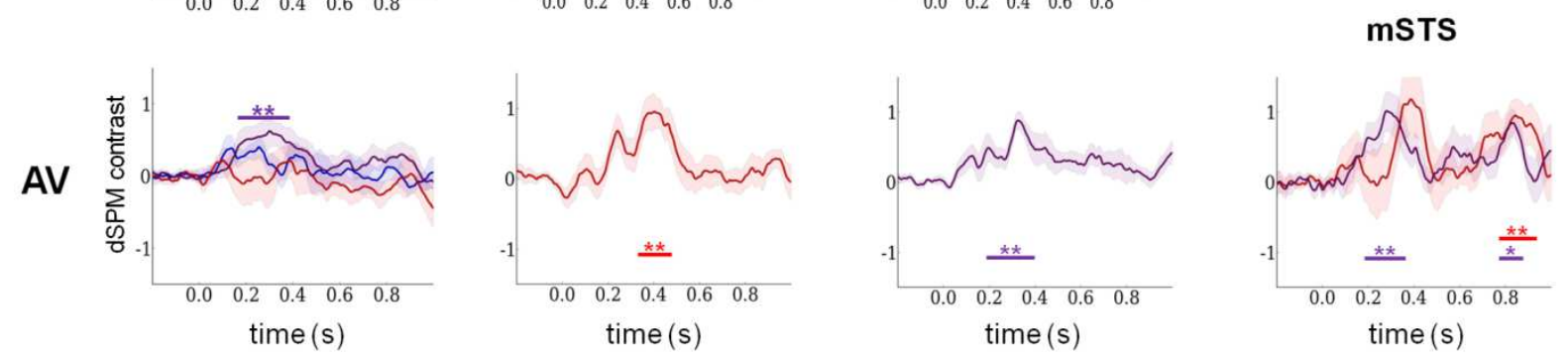

Figure 6. Functional selectivity in hMT+ and other regions of interest (ROIs) after V, AV and AVn

training. Visual coherence levels were classified into three groups according to participants' changes in perceptual thresholds, namely: "hard" coherence were those coherence levels that remain below the individual's post-training threshold (blue); "easy" coherence levels were those that remain above the pre-training threshold (red); "learned" coherence were those that went from below the perceptual threshold in pre-training to above the threshold of the participant after training (purple). Post- minus pre-training mean dSPM contrasts ( \pm 1 s.e.m.) are 
reported for all three groups (V: top; AVn: middle; AV: bottom). In hMT+, all categories are reported while in other ROIs only categories with significant differences are shown for clarity. Strikingly, only AV presented a significant difference in hMT+ observed as an increase of amplitude of the response to the 'learned' coherence levels. When considering all other ROIs defined in Fig. 2B, only AV presented significant time clusters for the 'learned' category in right mSTS while all groups presented significant increases in response to the 'easy' category in ITC. The analysis was extended to bilateral ventro-lateral PreFrontal Cortex (vlPFC) which strikingly revealed significant time clusters for all three groups but solely for the learned coherence levels. Significant clusters were determined using a pairwise cluster permutation algorithm and are indicated below the curves with bars (cf. Table 1). ‘*’: corrected p values inferior to 0.05 , ‘**': corrected $\mathrm{p}$ values inferior to 0.01 .

\begin{tabular}{|c|c|c|c|}
\hline \multirow[t]{2}{*}{ ROI } & $\mathbf{V}$ & $\mathbf{A V}$ & AVn \\
\hline & \multicolumn{3}{|c|}{ LEARNED category } \\
\hline hMT+ & n.s. & $\begin{array}{c}160: 390 \mathrm{~ms} \\
\mathrm{p}=0.0059\end{array}$ & n.s. \\
\hline mSTS & n.s. & $\begin{array}{c}180: 360 \mathrm{~ms} \\
\mathrm{p}=0.0088 \\
770: 880 \mathrm{~ms} \\
\mathrm{p}=0.019\end{array}$ & n.s. \\
\hline \multirow[t]{2}{*}{ vlPFC } & $\begin{array}{c}260: 390 \mathrm{~ms} \\
\mathrm{p}=0.0019 \\
550: 680 \mathrm{~ms} \\
\mathrm{p}=0.0054\end{array}$ & $\begin{array}{c}190: 390 \mathrm{~ms} \\
\mathrm{p}=0.0044\end{array}$ & $\begin{array}{c}350: 510 \mathrm{~ms} \\
\mathrm{p}=0.0098\end{array}$ \\
\hline & \multicolumn{3}{|c|}{ EASY category } \\
\hline ITC & $\begin{array}{c}250: 410 \mathrm{~ms} \\
\mathrm{p}=0.0064\end{array}$ & $\begin{array}{c}330: 480 \mathrm{~ms} \\
\mathrm{p}=0.0054\end{array}$ & $\begin{array}{c}380: 610 \mathrm{~ms} \\
\mathrm{p}=0.0029\end{array}$ \\
\hline mSTS & n.s. & $\begin{array}{c}770: 930 \mathrm{~ms} \\
\mathrm{p}=0.0068\end{array}$ & n.s. \\
\hline
\end{tabular}

Table 1. Summary of significant clusters observed in Figure 6. Latencies and corrected p values are provided for each ROIs (rows) and for each training group (columns).

Considering that hMT+ did not present selective changes to the 'learned' coherence levels notably in the $\mathrm{V}$ and $\mathrm{AVn}$ groups, we asked whether other cortical regions could significantly contribute to the obtained perceptual improvements. To this aim, neural responses in the observed regions of interest (ROIs, Figure 2B) were quantified and contrasted in pre- and post-training as a function of the defined perceptual categories (Fig. 6).

\subsection{Extended selectivity to other ROIs}


As previously done for $\mathrm{hMT}+$, contrasts of post- minus pre-training were separately tested for each training group and for each perceptual category by using a pairwise cluster permutation algorithm. For clarity, only those ROIs and time courses presenting significant differences are reported in Fig. 6 and a summary of significant cluster values and latencies is also provided in Table 1.

First, and common to all three groups, a significant response increase in post-training was observed in ITC but solely for the 'easy' category. Interestingly, different latencies were noticeable in each group (Fig.6, second column): $\sim 250$ to $410 \mathrm{~ms}$ in the V group, $\sim 330$ to 480 ms in the AV group and $\sim 380$ to $610 \mathrm{~ms}$ in the AVn group. This pattern suggests that in this task, color-motion binding may have equally improved in all participants irrespective of their training but solely when coherence discrimination was easiest. No significant differences were otherwise seen for any other perceptual categories in these ROIs.

As no other significant changes for the 'learned' category were seen in all ROIs to account for $\mathrm{V}$ and AVn perceptual improvements, we added a selection criterion for our analysis.

Specifically, several lines of research have shown that the lateral prefrontal cortex is a major site of convergence for the dorsal and ventral visual (Ungerleider and Mishkin, 1982) and auditory (Rauschecker and Tian, 2000) streams but also an important site of multisensory convergence (Romanski, 2004, 2007; Romanski and Hwang, 2012). We thus extended our analysis to bilateral vlPFC using Freesurfer neuroanatomical parcellations to define the ROI. Strikingly, significant time clusters were found in this region specifically for the 'learned' category and for all three groups (Fig. 6, third column). Two significant clusters were seen in V spanning 260 to $390 \mathrm{~ms}$ and 550 to $680 \mathrm{~ms}$; one surprisingly early significant cluster was seen in AV spanning 190 to $390 \mathrm{~ms}$ and one significant cluster in AVn spanning 350 to 510 ms. In addition, the AV group (Figure 6, fourth column) was the only group which presented 
a significant response increase in both the learned and the easy category in right $\mathrm{mSTS}$ at late latencies (770 to $930 \mathrm{~ms}$ ) but also, and crucially, significant changes for the 'learned' category at the same latencies as in hMT+ (i.e. $\sim 200$ to $\sim 400 \mathrm{~ms}$ ).

Altogether, these results strongly suggest that the boost in sensitivity observed in hMT+ may not result from local plasticity but from the engagement of a larger network in the computations of color-motion binding and coherence discrimination including prefrontal regions.

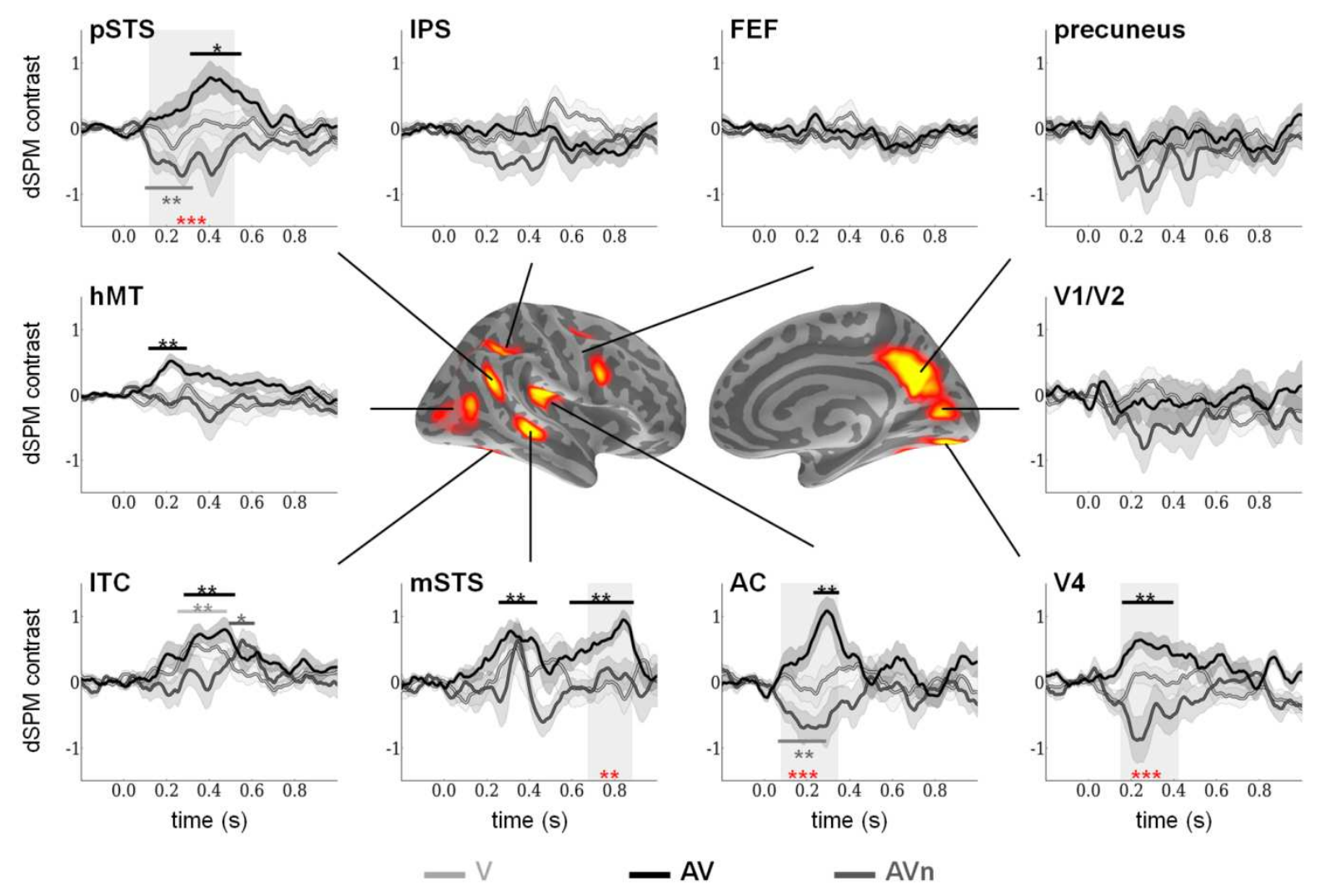

Figure 7. Main effects of training in all three groups across all coherence levels. Post- minus pre-training contrasts of mean current source estimates (dSPM, \pm 1 s.e.m.) across all RDK coherence levels and for each region of interests (see Fig. 2). Differential time series are reported in light grey for V, in black for AV and in dark grey for AVn. The effect of training in a given group was tested with a two-tailed paired t-test combined with a cluster permutation algorithm: significant differences are indicated with light grey bars (V), black bars $(\mathrm{AV})$ and dark grey bars (AVn). In $\mathrm{V}$, main effects of training irrespective of coherence levels can be seen in ITC from 200 to $400 \mathrm{~ms}$ post-coherence onset. In AV, main effects are seen in several regions including hMT+, ITC, mSTS, V4, pSTS and AC. In AVn, main effects are seen in ITC, pSTS, and AC. In order to test the main effects of training type (V, AV or AVn) irrespective of coherence levels, a F-test was performed in combination with a cluster permutation algorithm for all ROIs. The shaded areas highlight the latencies of significant 
differences between the training groups; red stars indicate the corresponding degree of significance. As can be seen, four main regions capture the main differences across the three training groups, namely: middle and posterior STS, V4 and AC. * corrected p values inferior to 0.05 ; ** corrected p values inferior to 0.01 ; *** corrected $\mathrm{p}$ value inferior to 0.001 .

\begin{tabular}{|c|c|c|c|c|}
\hline \multicolumn{5}{|c|}{ pre- vs. post-training - all coherence levels } \\
\hline & & t-tests & & F-tests \\
\hline ROI & $\mathbf{V}$ & AV & AVn & $\mathbf{V}, \mathbf{A V}, \mathbf{A V n}$ \\
\hline hMT+ & n.s. & $\begin{array}{c}130: 290 \mathrm{~ms} \\
\mathrm{p}=0.0044\end{array}$ & n.s. & n.s. \\
\hline mSTS & n.s. & $\begin{array}{c}250: 440 \mathrm{~ms} \\
\mathrm{p}=0.0083 \\
600: 900 \mathrm{~ms} \\
\mathrm{p}=0.0015\end{array}$ & n.s. & $\begin{array}{c}680: 880 \mathrm{~ms} \\
\mathrm{p}=0.0055\end{array}$ \\
\hline pSTS & n.s. & $\begin{array}{c}320: 560 \mathrm{~ms} \\
\mathrm{p}=0.016\end{array}$ & $\begin{array}{c}120: 320 \mathrm{~ms} \\
\mathrm{p}=0.0078\end{array}$ & $\begin{array}{c}120: 520 \mathrm{~ms} \\
\mathrm{p}=0.0007\end{array}$ \\
\hline V4 & n.s. & $\begin{array}{c}160: 400 \mathrm{~ms} \\
\mathrm{p}=0.0068\end{array}$ & n.s. & $\begin{array}{c}150: 420 \mathrm{~ms} \\
\mathrm{p}=0.00095\end{array}$ \\
\hline ITC & $\begin{array}{c}260: 500 \mathrm{~ms} \\
\mathrm{p}=0.007\end{array}$ & $\begin{array}{c}300: 540 \mathrm{~ms} \\
\mathrm{p}=0.0049\end{array}$ & $\begin{array}{c}500: 630 \mathrm{~ms} \\
\mathrm{p}=0.029\end{array}$ & n.s. \\
\hline $\mathbf{A C}$ & n.s. & $\begin{array}{c}210: 340 \mathrm{~ms} \\
\mathrm{p}=0.0088\end{array}$ & $\begin{array}{l}60: 280 \mathrm{~ms} \\
p=0.0049\end{array}$ & $\begin{array}{l}80: 340 \mathrm{~ms} \\
p=0.00075\end{array}$ \\
\hline
\end{tabular}

Table 2. Summary of significant clusters observed in Figure 7. Latencies and corrected p values are provided for each ROIs (rows) and for each training group (columns).

\subsection{A larger network distinctively dissociate the three training groups}

We now ask whether a non-selective training effect can be observed irrespective of the RDK coherence levels across all three groups, thereby reflecting an overall effect of task improvement. Similar to previous analyses, the evoked responses elicited by the presentation of all RDK coherence levels were grand-averaged, source reconstructed and averaged within each ROI as defined in Fig. 2.

The time courses in pre- and post-training are illustrated in Supplementary Figures S3 and S4, respectively. With the exception of visual area V4, no significant differences were observed between the three groups before training (Supp. Fig. S3); in post-training, the time courses across the three groups significantly differed only in right mSTS (Supp. Fig. S4). The source amplitudes in the different ROIs were then contrasted between the pre- and post-training blocks and tested with a cluster permutation algorithm in each group (Fig. 7 and Table 2). 
First, all three groups presented a main effect of training in ITC corresponding to positive clusters at increasing latencies, namely in V: 260 to $500 \mathrm{~ms}$; in AV: 300 to $540 \mathrm{~ms}$ and in AVn: 500 to $630 \mathrm{~ms}$. Second, no additional effects were found for the V group. Third in the AV group, a large network was observed revealing significant post-training responses increase in hMT+ (130 to $290 \mathrm{~ms}$ post-coherence onset), in right mSTS with two temporal clusters (250 to $440 \mathrm{~ms}$ and 600 to $900 \mathrm{~ms}$ ) post-coherence onset, in V4 (160 to $400 \mathrm{~ms}$ ), in pSTS (320 to $560 \mathrm{~ms}$ ) and in AC (210 to $340 \mathrm{~ms}$ ). Fourth and interestingly, pSTS and AC presented opposite effects for AVn, with a significant decrease of activity in post-training for latencies of 120 to $320 \mathrm{~ms}$ in pSTS and of 60 to $280 \mathrm{~ms}$ in AC.

In order to directly contrast the three training groups, an F-test was combined with a cluster permutation algorithm: the earliest effect was observed in AC starting at 80 ms postcoherence onset (and lasting $260 \mathrm{~ms}$ ), rapidly followed by a long sustained differentiation in pSTS from 120 to $520 \mathrm{~ms}$ and in V4 from 160 and $400 \mathrm{~ms}$; a late main effect was observed in the right mSTS from 680 to $880 \mathrm{~ms}$. 

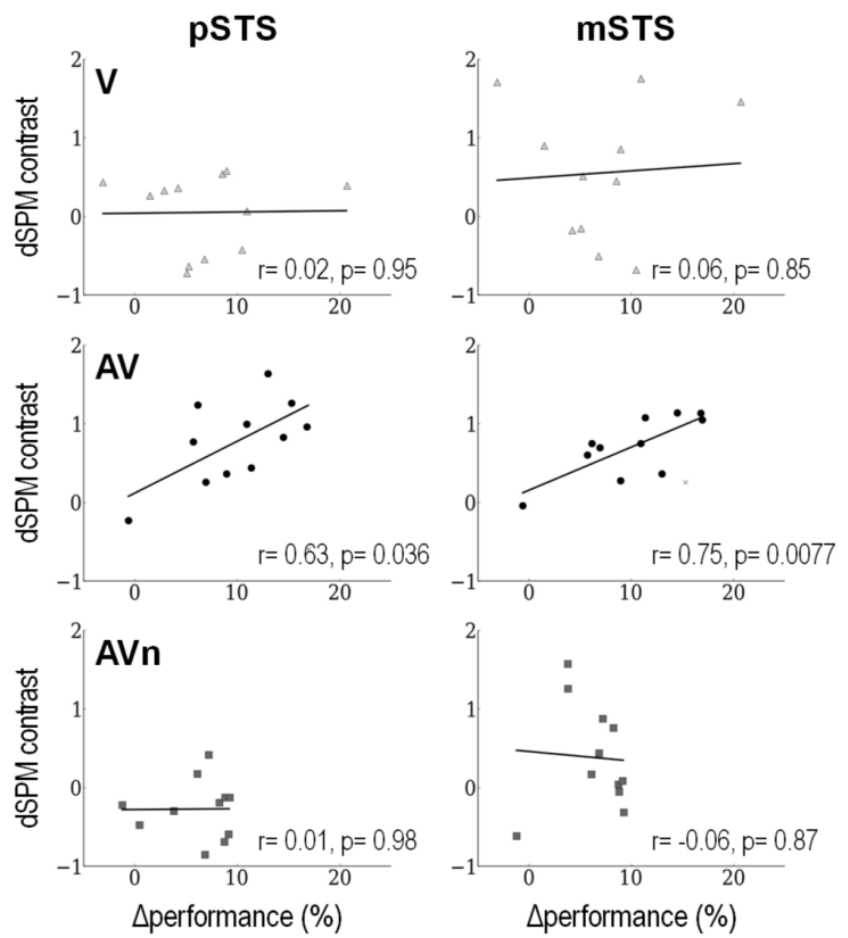

Figure 8. Main effects of training in bilateral pSTS and right mSTS are uniquely observed in the AV group. Mean dSPM contrasts in bilateral pSTS (left column) and right mSTS (right column) as a function of individuals' mean performance increases over all coherence levels in $\mathrm{V}$ (top), $\mathrm{AV}$ (middle) and AVn (bottom). dSPM contrasts were computed by collapsing all RDK coherence levels and averaged over the time windows corresponding to significant differences in AV (i.e. over 320-560 ms in pSTS and 250-440 ms in mSTS) as reported in Figure 7. Significant positive correlations between overall performance and source estimate amplitude were observed solely in the AV group specifically in pSTS $(r=0.63, p=0.036)$ and in $\operatorname{mSTS}(r=$ $0.75, \mathrm{p}=0.0077)$. ' $\mathrm{x}$ ': automatically detected outliers.

To better comprehend the role of mSTS and pSTS, the post- minus pre- contrasts of source estimate amplitudes were plotted as a function of post- minus pre- performance separately for each group (Figure 8). A significant correlation was observed in both ROIs but again, solely for the AV group. This result suggests that while mSTS and pSTS are not selective to the RDK coherence levels, these regions play a significant role in the task improvements observed in the AV group but not in the other groups. Altogether, our results highlight the distinct contribution of different cortical areas either selective to the RDK coherence levels or to the training history of the participant. A summary and working hypothesis is provided in 
Figure 9 on the functional role of the ROIs contribution to perceptual improvements observed in the three groups of participants.

A
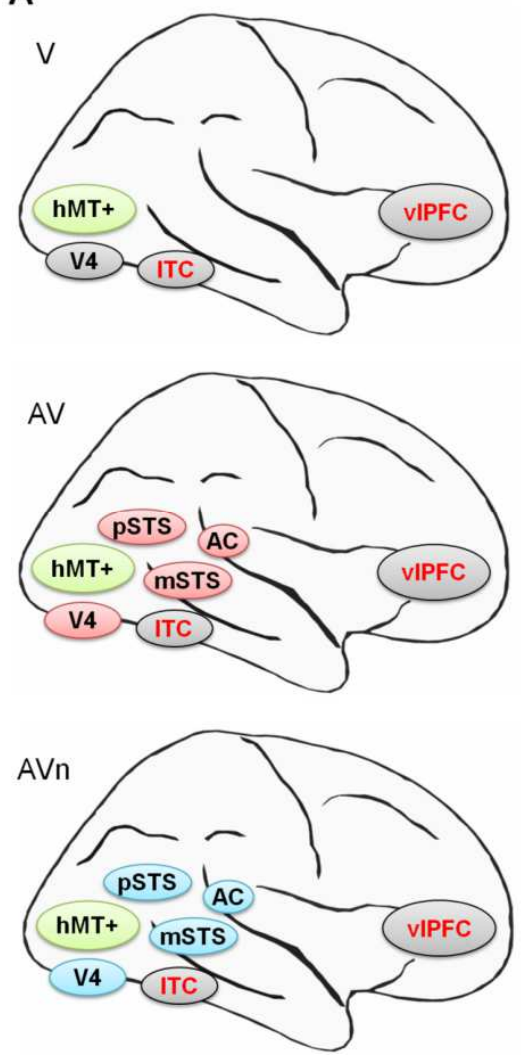

B
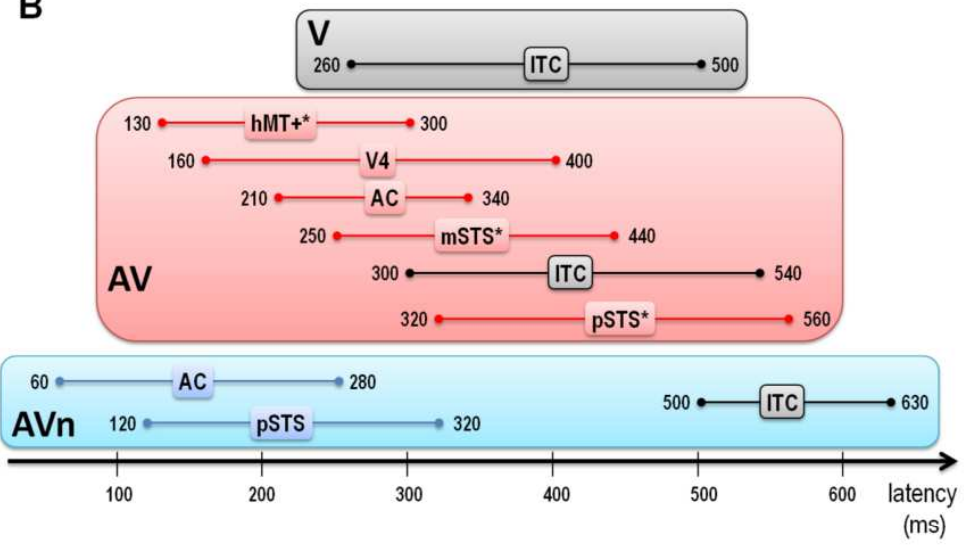

C

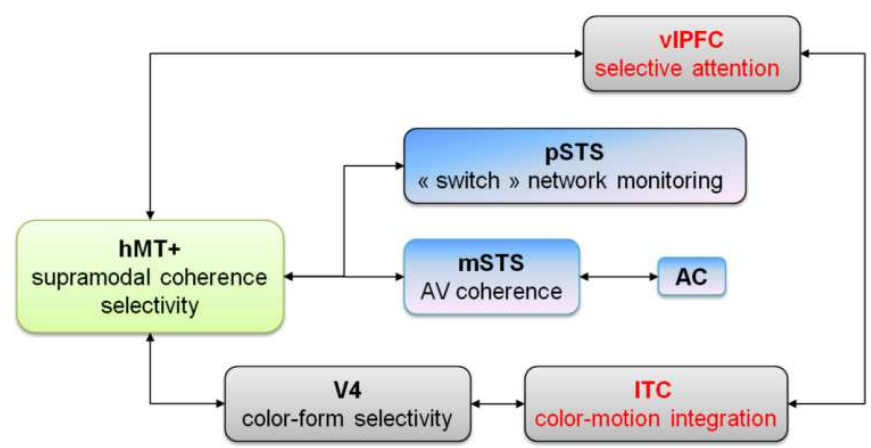

Figure 9. A working hypothesis for supramodal processing and reverse hierarchy plasticity. Panel A:

Synthetic illustration of ROIs showing significant post-training changes in neural responses after training in the $\mathrm{V}, \mathrm{AVn}$ and AV groups. Significant changes in hMT+, V4, ITC and vlPFC were common to all three groups whereas pSTS, mSTS, and AC were specific to the multisensory AV and AVn groups. The network observed in post-multisensory training was thus more extensive than in visual training. Strikingly, the pattern of activation in the control AVn group and in the AV group was notably reversed in several regions including pSTS, AC, mSTS and V4: this suggests selective modulations of these cortical regions based on the stimuli presented during training. Panel B: Synthetic summary of latency of main significant effects obtained when contrasting post- vs. pre-training data irrespective of coherence levels. The temporal overlaps of significant effects over the extensive network observed in the $\mathrm{AV}$ trained groups suggest a complex pattern of communication between these brain regions. Importantly, a major latency difference can be observed between the AV and AVn groups in AC and pSTS with an early decreased activity in these regions in the AVn group and a later increase activity in the AV group. We contend that this difference is consistent with pSTS acting as a «switch » allowing the implication of mSTS in the computation of coherence motion. Panel C: A basic hypothesis for the functional network implicated in visual learning in the $\mathrm{V}, \mathrm{AV}$ and $\mathrm{AVn}$ groups. The distinctive pattern of cortical activity that significantly dissociated the three training groups was a significant increase and decrease of activity in AV and $\mathrm{AVn}$, respectively for the pSTS, mSTS, AC and V4, suggesting direct functional connectivity between these regions (blue/red boxes). No significant changes of activity were observed in these regions for the $\mathrm{V}$ group. 
Common to all three training groups, hMT+ and vlPFC showed discriminable cortical responses as a function of the learned coherence levels. Additionally, all three groups showed an increased activity in ITC only for the easy coherence levels suggesting an improvement in color-motion binding. In hMT+, the increase spread of neural response was shared by $\mathrm{V}$ and the control AVn, whereas selective activity was seen solely for the AV group. Altogether, our results suggest a regulation of hMT+ activity by upstream computations notably in the AV and AVn groups. The specific implications of V1/V2, IPS and FEF (ROIs obtained in Fig. 2) remain to be determined.

\section{DISCUSSION}

In this study, we asked whether learning to discriminate visual coherent motion would rapidly benefit from hearing matched acoustic features. To this end, three groups of participants underwent training with visual (V), correlated (AV) or arbitrary (AVn) audiovisual pairings while being recorded with MEG. First, all three groups showed a significant decrease of their visual coherence discrimination thresholds after a very short training. However, AV participants significantly outperformed participants in the V and AVn groups although V participants were the only ones showing a significant increase in confidence rating. Second, all three groups showed a common activation pattern in two distinct cortical regions (ITC and vlPFC): a comparable post-training increase of neural activity in the ventral visual stream (ITC) suggests that color-motion binding consistently improved when coherence discrimination was easily achieved. One interpretation is that easy detection of coherent motion allowed for more efficient motion-color binding. All three groups showed increased post-training activity in vIPFC specifically for the learned coherence levels, suggesting a strong and selective implication of prefrontal cortex in learning. Conversely, distinct patterns of activity distinguished the three groups of participants with the multisensory trained groups (AV and AVn) showing an opposite pattern of post-training activity in a network comprising pSTS, mSTS, and AC (cf. Figure 9). This suggests that multisensory training fundamentally altered the network implicated in the analysis of visual coherent motion stimuli and that a uni- 
$v s$. a multi-sensory training can selectively shape the activity of the implicated network.

Third, and crucially, AV participants were the only group showing a post-training gain of selectivity in hMT+ as captured by a significant shift in their neurometric thresholds.

Altogether, we thus interpret our results as evidence for supramodal processing elicited by the presentation of coherent audiovisual features during training. Our results suggest that supramodal processing allowed the fine-tuning of downstream selectivity in visual cortices in agreement with the reverse hierarchy hypothesis (Ahissar and Hochstein, 2004; Proulx et al., 2012); if this hypothesis is correct, multisensory training can open new empirical venues for the understanding of top-down plasticity in implicit perceptual learning and greatly speed up the use of sensory-substitution devices in sensory-impaired population.

\subsection{Supramodal objects and cross-sensory feature matching}

Statistical contingencies across sensory modalities can be learned (Seitz et al., 2007; Mitchel and Weiss, 2011) and multisensory information has been shown to benefit perceptual learning (e.g. Shams and Seitz, 2008; for an extensive review, see Proulx et al., 2012). However, the observed perceptual improvements are generally small and can require a long training time: with ten days of training, presenting auditory motion cues has been shown to improve visual direction discrimination (Seitz et al., 2006) and acoustic cues can alter the direction of visual motion (Freeman and Driver, 2008; Hidaka et al., 2011). Here, consistent with the hypothesis that using redundant multisensory information should yield greater benefits (Alais and Burr, 2004), we capitalized on cross-sensory feature matching, namely the temporal coherence between auditory spectral changes and visual spatial patterning over time. The temporal coherence of audiovisual information is inherent to natural stimuli: in particular, the envelope of auditory speech is known to correlate with the speaker's facial gestures (Grant and Seitz, 
2000; Grant and Greenberg, 2001; Schwartz et al., 2004; Chandrasekaran et al., 2009) and more generally, auditory pitch and visual spatial frequency undergo automatic cross-sensory matching (Maeda et al., 2004; Evans and Treisman, 2010). The comodulation of audiovisual signals is thus a fundamental attribute of natural scenes that enables the brain to appropriately bind sensory features belonging to the same physical object, albeit processed through different sensory processing streams. Hence, by using matched audiovisual correspondences, we expected rapid cross-sensory mapping allowing for efficient learning in the AV group as compared to the control AVn and the V groups. In agreement with this hypothesis, the AV group significantly outperformed the $\mathrm{V}$ and the control AVn groups, suggesting that the mere presence of sound is not sufficient to improve visual coherence discrimination and rather, that the correlated temporal structure imposed on the audiovisual stimuli during training largely benefitted visual discrimination and did so in a very short exposure time.

\subsection{Implicit learning}

An additional intriguing feature was that unlike $\mathrm{V}$ learners, the confidence rating of the AV and AVn groups did not change after learning. Confidence ratings are a well-established means to assess conscious knowledge in decision making (e.g. Dienes, 2008) and have recently been argued to be most reliable in assessing the lowest level of subjective awareness (Wierzchoń et al., 2012). The lack of increased confidence rating in participants undergoing multisensory training strongly suggests that cross-sensory mapping occurred at an implicit level during training, which is consistent with the notion of automatic binding in multisensory integration (Talsma et al., 2010; Kösem and van Wassenhove, 2012) and with prior report of unconscious transfer between auditory and visual sensory modalities (Levy-Tzedek et al., 
2012b). These results indicate that no cognitive strategy was used by participants trained in multisensory conditions to accomplish the task. One possibility is that differences in confidence ratings may be accounted for by inter-individual differences irrespective of learning (Song et al., 2011). The dissociation of subjective awareness observed here - i.e. improved performance without improved confidence rating - could be tentatively explained by the nature of what has been learned: the response pattern observed in ITC suggests that color-motion binding has improved in all participants irrespective of their training whereas visual coherence discrimination was solely observed in the AV group. Additionally, the preand post-training perceptual thresholds specifically focused on data collected in visual alone conditions in all three groups, thereby alleviating the possibility of divided attentional effects in task performance.

\subsection{Supramodal object representation in vlPFC?}

As previously mentioned, the audiovisual stimuli used during training were specifically designed to mimic the correspondences of auditory and visual attributes predicted from natural communication stimuli such as speech and monkey vocalizations although we arguably avoided possible overt semantic categorizations (face, speech). These audiovisual features rely on the correlated temporal structuring of acoustic and visual information and focused on the spectrotemporal attributes of the signals requiring color-motion binding for overt response ("red (green) RDK is most coherent"). Hence, during training (data not reported), the matching between visual and acoustic features would likely be comparable to the one taking place in the context of natural stimuli. 
In her recent review, Chan (Chan, 2013) contrasts the evidence in favor of a domain general $v s$. a domain specific contribution of vlPFC and suggests that vlPFC primarily represents object-feature information. In our study, a possible interpretation for the selective activation to the learned coherence levels observed in vlPFC (Fig. 6) irrespective of training groups may be the increased representational salience of supramodal coherence, namely, the combined (auditory and/or visual) features enabling the neural representation of a "coherent object" irrespective of its color or direction of motion - hence, supramodal coherence. In the context of learning, the enhanced activation may be relevant by virtue of binding across visual and/or auditory streams specifically for those levels of coherences newly recognized. vlPFC is a known site of convergence for the dorsal and visual streams of both auditory and visual systems and a major site of convergence for the representation of multisensory information (Romanski et al., 1999; Romanski, 2007; Romanski and Hwang, 2012). Interestingly, vlPFC has also been implicated in the representation of communication signals in monkey recordings (Sugihara et al., 2006) suggesting that this region is particularly well-suited for the computations of natural and matched cross-sensory stimuli such as the ones utilized here. These results are further consistent with several neuroimaging studies showing the implication of vlPFC for semantic retrieval and response selection in the context of multisensory processing (e.g. Werner and Noppeney, 2010).

\subsection{Functional selectivity of hMT+ : psycho- and neuro-metric thresholds}

Although previous studies have reported activation of hMT + to the presentation of auditory stimuli (Poirier et al., 2005, 2006) and matched audiovisual motion (Alink et al., 2008; Scheef et al., 2009; von Saldern and Noppeney, 2013), the evidence for auditory motion processing 
in this region is scarce: from a neurophysiological standpoint, Ilg and Churan (Ilg and Churan, 2004) have shown that the presentation of visual and audiovisual motion elicits the same neural response in motion area MT but no significant response to the presentation of auditory motion alone was observed in this region. Hence, the most convincing evidence for the capabilities of hMT + to compute motion processing supramodally - i.e. irrespective of the sensory modality of inputs - comes from studies of sensory-impaired and blind populations (Morrone, 2010; Voss and Zatorre, 2012; Ricciardi et al., 2013) in which functional recycling can readily be observed for the benefit of other sensory modalities.

One study (Bedny et al., 2010) has notably suggested the existence of a sensitive period around 2 years of age for the acquisition of visual functional selectivity in this region; additionally, the lack of exposure to visual information was shown to prevent visual selectivity in this region although hMT+ in late blind populations can be functionally recycled to the benefit of auditory motion processing. In this context, we asked whether a shorttraining capitalizing on cross-sensory matching could benefit plasticity in this region. In particular, comprehensive reviews have recently suggested that hMT+ could benefit from topdown processing as a major means to achieve supramodal selectivity (Morrone, 2010; Proulx et al., 2012).

One crucial result of our study is that in healthy individuals, selectivity in hMT+ can significantly benefit from correlated audiovisual sensory inputs during training. By means of neurometric characterization of MEG signals in $\mathrm{hMT}+$, we showed that after a short training, neural plasticity in this cortical region was solely achieved in the AV group not in the V or the AVn group. Hence, the direct comparison of perceptual discrimination and neurometric thresholds suggest that although all three groups performed better after training, only the AV group showed a significant change in neurometric threshold and thus conservatively displayed perceptual learning and plasticity (Goldstone, 1998; Gilbert et al., 2001; Fahle, 2005; Seitz 
and Watanabe, 2005). This observation is particularly relevant in complementing a recent discussion on the interpretation of psychometric thresholds in perceptual learning studies (Gold and Ding, 2013).

Additional analyses conducted on the datasets obtained during training will shed light on the specific contribution of auditory information during audiovisual processing and the integrative mechanisms leading to the differentiation of the network in the multisensory trained groups. The changes in neurometric thresholds observed in hMT+ are particularly puzzling in light of the recent lack of evidence for neurometric threshold or slope changes after training in this region (Gold et al., 2010). Below, we extend our discussion on the selective network dynamics that was shown to dissociate the three training groups and elaborate a working hypothesis on the implication of supramodal processing for the top-down fine tuning of motion coherence processing in $\mathrm{hMT}+$.

\subsection{Reverse hierarchy and supramodal processing}

An extended network was seen in multisensory trained participants notably implicating pSTS, mSTS, and AC. Crucially, while activation increased in these regions in the AV group, activation decreased in these regions in the control AVn group. These areas showed no changes in the $\mathrm{V}$ group. This pattern of results shows that after training, identical visual stimuli are processed differently depending on the training history of participants even if the implication of vlPFC, ITC and V4 is preserved in all cases.

First, mSTS is characterized by a patchy organization of multisensory, auditory and visual selective neurons (Beauchamp et al., 2004a) and has systematically been implicated in the analysis of multisensory timing with possible feedback to sensory cortices (Noesselt et al., 
2007). In post-training data, pSTS and mSTS correlated with the individual improvements in the AV group: one possible interpretation is that during training, mSTS processed coherent AV motion and transferred selectivity to hMT+ post-training. The modulation of hMT+ by mSTS could either enhance the salience of visual coherent motion during training (e.g. Lewis and Noppeney, 2010) or facilitate the extraction of task-relevant features for visual processing (Sasaki et al., 2010). Consistent with this interpretation, no mSTS activity was seen in the V group and decreased activity was seen in the AVn group.

Crucially then, the functional role of mSTS in post-training tests was preserved even in the absence of multisensory inputs: this suggests that plasticity implicating both uni- and multisensory neural populations found in mSTS occurred during AV and AVn training. However, the limited spatial resolution of MEG cannot disentangle the possible contribution of different neural populations in this region during or after training.

Second, pSTS has also been classically implicated in multisensory integration (Benevento et al., 1977; Bruce et al., 1981; Beauchamp et al., 2004a, 2004b; Lewis and Noppeney, 2010) and has recently been shown to mediate the temporal narrowing of audiovisual integration (Powers et al., 2012). Specifically, changes of effective connectivity between pSTS and downstream sensory regions have been reported after repeated presentations of temporally coincident audiovisual stimuli (Powers et al., 2012). pSTS is thus largely implicated in the temporal association of multisensory information but is also associated with the analysis of second-order visual motion (Noguchi et al., 2005) and biological motion (Saygin, 2007). Considering that post-training response patterns in pSTS were opposite in AV and AVn, this region may play the role of a "switch" by selectively enabling the communication of mSTS with the ventral visual stream. It is here crucial to note that the differences solely illustrate the training history of participants and not the mere presence or absence of AV stimulation. 
Overall, the latencies of the main effects were consistent with a feedback of information from temporal cortex to hMT+ (Figure 9). Specifically, the main effects of training irrespective of visual coherence levels (Fig. 9C) showed a clear sequencing and overlapping of differential activation in the multisensory trained group. The temporal overlapping of increased activation in pSTS, mSTS and auditory cortex with hMT+ in the AV group showed a strikingly different pattern compared to the sequencing observed in the AVn group (decreased activation of pSTS and AC). These results are consistent with the selective implication of pSTS and mSTS that were reported and the functional implication of pSTS as a switch enabling the flow of information from mSTS to hMT+ - thereby enabling or not the implication of multisensory regions in the computations of coherent motion.

In sum, we suggest that AV training favored supramodal computations of coherence in multisensory regions during training (mSTS) which remained engaged even in the absence of multisensory stimulation for the benefit of visual processing (hMT+) via pSTS (Fig. 9C). Previous studies have reported activation of hMT + to the presentation of auditory (Poirier et al., 2005) and matched audiovisual motion (Alink et al., 2008; Scheef et al., 2009); we thus extend these findings by showing a selective tuning of $\mathrm{hMT}+$ response to the presentation of coherent visual motion after AV training. In light of recent connectivity measures implicating pSTS (Powers et al., 2012), our results provide the first evidence for supramodal processing enabling reverse hierarchy of learning onto visual-specific areas (Ahissar and Hochstein, 2004; Morrone, 2010; Proulx et al., 2012). This scheme is consistent with the view that higher cortices may generalize learning and fine-tune downstream selectivity notably when considering the selectivity of vlPFC in all three groups (Ahissar and Hochstein, 1997, 2004). 


\section{CONCLUSIONS}

Our results suggest that the temporal structure of multisensory features can profoundly affect the analysis of sensory information and de facto implicate multisensory regions. This is consistent with several studies that have pointed out to early audiovisual interactions in sensory processing streams notably through the magnocellular system (Frassinetti et al., 2005; Schroeder and Foxe, 2005; Jaekl and Soto-Faraco, 2010) in line with neuroanatomical connectivity (Hackett et al., 2007). Importantly, our results suggest that the seminal spatiotemporal coincidence principle (Stein and Meredith, 1993; Meredith, 2002) is not only fundamental for supramodal processing but also critical in shaping up downstream neural selectivity of classically defined sensory specific areas. As such, the use of sensory features that naturally map across sensory modalities by virtue of temporal Gestalts principles provide a first step towards understanding the representation of multisensory invariance or supramodal objects in the brain. Practical implications of this research are foreseeable for the optimization of sensory substitution devices making use of natural cross-sensory mapping in audition, somatosensation and vision (Bach-y-Rita and Kercel, 2003; Amedi et al., 2007).

ACKNOWLEDGMENTS: This work was supported by a Marie Curie IRG-249222 and an ERC-YStG-263584 to V.vW and an ANR Schubert ANR-0909-JCJC-071 to P.C. We are grateful to the NeuroSpin nursing staff for their help in recruiting and preparing participants for MEG data acquisition.

\section{REFERENCES}

Ahissar M, Hochstein S (1997) Task difficulty and the specificity of perceptual learning. Nature 387:401-6 
Ahissar M, Hochstein S (2004) The reverse hierarchy theory of visual perceptual learning. Trends Cogn Sci 8:457-64

Ahlfors S, Simpson G, Dale A, Belliveau J, Liu A, Korvenoja A, Virtanen J, Huotilainen M, Tootell R, Aronen H, Ilmoniemi R (1999) Spatiotemporal activity of a cortical network for processing visual motion revealed by MEG and fMRI. J Neurophysiol 82:2545-55

Alais D, Burr D (2004) No direction-specific bimodal facilitation for audiovisual motion detection. Brain Res Cogn Brain Res 19:185-94

Alink A, Singer W, Muckli L (2008) Capture of auditory motion by vision is represented by an activation shift from auditory to visual motion cortex. J Neurosci 28:2690-7

Amano K, Goda N, Nishida S, Ejima Y, Takeda T, Ohtani Y (2006) Estimation of the timing of human visual perception from magnetoencephalography. J Neurosci 26:3981-91

Amedi A, Stern W, Camprodon J, Bermpohl F, Merabet L, Rotman S, Hemond C, Meijer P, Pascual-Leone A (2007) Shape conveyed by visual-to-auditory sensory substitution activates the lateral occipital complex. Nat Neurosci 10:687-9

Aspell J, Tanskanen T, Hurlbert A (2005) Neuromagnetic correlates of visual motion coherence. Eur J Neurosci 22:2937-45

Bach-y-Rita P, Kercel S (2003) Sensory substitution and the human-machine interface. Trends Cogn Sci 7:541-6

Bavelier D, Hirshorn E (2010) I see where you're hearing: how cross-modal plasticity may exploit homologous brain structures. Nat Neurosci 13:1309-11

Beauchamp M, Argall B, Bodurka J, Duyn J, Martin A (2004)(a) Unraveling multisensory integration: patchy organization within human STS multisensory cortex. Nat Neurosci $7: 1190-2$

Beauchamp M, Lee K, Argall B, Martin A (2004)(b) Integration of auditory and visual information about objects in superior temporal sulcus. Neuron 41:809-23

Bedny M, Konkle T, Pelphrey K, Saxe R, Pascual-Leone A (2010) Sensitive period for a multimodal response in human visual motion area MT/MST. Curr Biol 20:1900-6

Benevento L, Fallon J, Davis B, Rezak M (1977) Auditory-visual interaction in single cells in the cortex of the superior temporal sulcus and the orbital frontal cortex of the macaque monkey. Exp Neurol 57:849-72

Britten K, Shadlen M, Newsome W, Movshon J (1992) The analysis of visual motion $\square$ : a comparison of neuronal and psychophysical performance. J Neurosci 12:4745-65

Bruce C, Desimone R, Gross C (1981) Visual properties of neurons in a polysensory area in superior temporal sulcus of the macaque. J Neurophysiol 46:369-84 
Chan A (2013) Functional organization and visual representations of human ventral lateral prefrontal cortex. Front. Psychol. 4:371

Chandrasekaran C, Trubanova A, Stillittano S, Caplier A, Ghazanfar A (2009) The natural statistics of audiovisual speech. PLoS Comput Biol 5:e1000436

Dale A, Fischl B, Sereno M (1999) Cortical surface-based analysis. I. Segmentation and surface reconstruction. Neuroimage 9:179-94

Dale A, Liu A, Fischl B, Buckner R (2000) Dynamic statistical parametric mapping: combining fMRI and MEG for high-resolution imaging of cortical activity. Neuron 26:55-67

Dienes Z (2008) Subjective measures of unconscious knowledge. Prog Brain Res 168:49-64

Dormal G, Collignon O (2011) Functional selectivity in sensory-deprived cortices. J

Neurophysiol 105:2627-30

Driver J, Spence C (2000) Multisensory perception: beyond modularity and convergence. Curr Biol 10:R731-5

Evans K, Treisman A (2010) Natural cross-modal mappings between visual and auditory features. J Vis 10:1-12

Fahle M (2005) Perceptual learning: specificity versus generalization. Curr Opin Neurobiol $15: 154-60$

Fischl B, Dale A (2000) Measuring the thickness of the human cerebral cortex from magnetic resonance images. Proc Natl Acad Sci USA 97:11050-5

Fischl B, Sereno M, Dale A (1999)(a) Cortical surface-based analysis. II: inflation, flattening, and a surface-based coordinate system. Neuroimage 9:195-207

Fischl B, Sereno M, Tootell R, Dale A (1999)(b) High-resolution intersubject averaging and a coordinate system for the cortical surface. Hum Brain Mapp 8:272-84

Frassinetti F, Bolognini N, Bottari D, Bonora A, Làdavas E (2005) Audiovisual integration in patients with visual deficit. J Cogn Neurosci 17:1442-52

Freeman E, Driver J (2008) Direction of visual apparent motion driven solely by timing of a static sound. Curr Biol 18:1262-6

Ghazanfar A, Schroeder C (2006) Is neocortex essentially multisensory? Trends Cogn Sci $10: 278-85$

Gilbert C, Sigman M, Crist R (2001) The neural basis of perceptual learning. Neuron 31:68197

Gold J, Ding L (2013) How mechanisms of perceptual decision-making affect the psychometric function. Prog. Neurobiol. 103:98-114 
Gold J, Law C, Connolly P, Bennur S (2010) Relationships between the threshold and slope of psychometric and neurometric functions during perceptual learning: implications for neuronal pooling. J Neurophysiol 103:140-54

Goldstone R (1998) Perceptual learning. Annu. Rev. Psychol 49:585-612

Gramfort A, Luessi M, Larson E, Engemann D, Strohmeier D, Brodbeck C, Goj R, Jas M, Brooks T, Parkkonen L, Hämäläinen M (2013)(a) MEG and EEG data analysis with MNEPython. Front Neuroinform 7:42

Gramfort A, Luessi M, Larson E, Engemann D, Strohmeier D, Brodbeck C, Parkkonen L, Hämäläinen M (2013)(b) MNE software for processing MEG and EEG data. Neuroimage

Grant K, Greenberg S (2001) Speech intelligibility derived from asynchronous processing of auditory-visual information. In Proc Conf Audio-Visual Speech Processing Aalborg, Denmark, p. 132-7. Available at: http://www.iscaspeech.org/archive_open/avsp01/av01_132.html [Accessed December 11, 2013].

Grant K, Seitz P (2000) The use of visible speech cues for improving auditory detection of spoken sentences. J Acoust Soc Am 108:1197-208

Gross J, Baillet S, Barnes G, Henson R, Hillebrand A, Jensen O, Jerbi K, Litvak V, Maess B, Oostenveld R, Parkkonen L, Taylor J, Wassenhove V van, Wibral M, Schoffelen J (2012) Good practice for conducting and reporting MEG research. Neuroimage 65:349-63

Hackett T, La Mothe L De, Ulbert I, Karmos G, Smiley J, Schroeder C (2007) Multisensory convergence in auditory cortex, II. Thalamocortical connections of the caudal superior temporal plane. J Comp Neurol 502:924-52

Hämäläinen M, Sarvas J (1989) Realistic conductivity geometry model of the human head for interpretation of neuromagnetic data. IEEE Trans Biomed Eng 36:165-71

Händel B, Lutzenberger W, Thier P, Haarmeier T (2007) Opposite dependencies on visual motion coherence in human area MT+ and early visual cortex. Cereb Cortex 17:1542-9

Hidaka S, Teramoto W, Sugita Y, Manaka Y, Sakamoto S, Suzuki Y (2011) Auditory motion information drives visual motion perception. PLoS One 6:e17499

Ilg U, Churan J (2004) Motion perception without explicit activity in areas MT and MST. J Neurophysiol 92:1512-23

Jaekl P, Soto-Faraco S (2010) Audiovisual contrast enhancement is articulated primarily via the M-pathway. Brain Res 1366:85-92

Kösem A, Wassenhove V van (2012) Temporal structure in audiovisual sensory selection. PLoS One 7:e40936

Lam K, Kaneoke Y, Gunji A, Yamasaki H, Matsumoto E, Naito T, Kakigi R (2000) Magnetic response of human extrastriate cortex in the detection of coherent and incoherent motion. Neuroscience 97:1-10 
Levy-Tzedek S, Hanassy S, Abboud S, Maidenbaum S, Amedi A (2012)(a) Fast, accurate reaching movements with a visual-to-auditory sensory substitution device. Restor Neurol Neurosci 30:313-23

Levy-Tzedek S, Novick I, Arbel R, Abboud S, Maidenbaum S, Vaadia E, Amedi A (2012)(b) Cross-sensory transfer of sensory-motor information: visuomotor learning affects performance on an audiomotor task, using sensory-substitution. Sci Rep 2:949

Lewis R, Noppeney U (2010) Audiovisual synchrony improves motion discrimination via enhanced connectivity between early visual and auditory areas. J Neurosci 30:12329-39

Lin F, Belliveau J, Dale A, Hämäläinen M (2006) Distributed current estimates using cortical orientation constraints. Hum Brain Mapp 27:1-13

Lomber S, Meredith M, Kral A (2010) Cross-modal plasticity in specific auditory cortices underlies visual compensations in the deaf. Nat Neurosci 13:1421-7

Maeda F, Kanai R, Shimojo S (2004) Changing pitch induced visual motion illusion. Curr Biol 14:R990-1

Mao Y, Hua T, Pallas S (2011) Competition and convergence between auditory and crossmodal visual inputs to primary auditory cortical areas. J Neurophysiol 105:1558-73

Maris E, Oostenveld R (2007) Nonparametric statistical testing of EEG- and MEG-data. J Neurosci Methods 164:177-90

Maruyama K, Kaneoke Y, Watanabe K, Kakigi R (2002) Human cortical responses to coherent and incoherent motion as measured by magnetoencephalography. Neurosci Res 44:195-205

Meijer P (1992) An experimental system for auditory image representations. IEEE Trans Biomed Eng 39:112-21

Melara R, O’Brien T (1987) Interaction between synesthetically corresponding dimensions. J Exp Psychol Gen 116:323-36

Mercier M, Schwartz S, Michel C, Blanke O (2009) Motion direction tuning in human visual cortex. Eur J Neurosci 29:424-34

Meredith M (2002) On the neuronal basis for multisensory convergence: a brief overview. Brain Res Cogn Brain Res 14:31-40

Mitchel A, Weiss D (2011) Learning across senses: cross-modal effects in multisensory statistical learning. J Exp Psychol Learn Mem Cogn 37:1081-91

Morrone M (2010) Brain development: critical periods for cross-sensory plasticity. Curr Biol 20:R934-6

Morrone M, Tosetti M, Montanaro D, Fiorentini A, Cioni G, Burr D (2000) A cortical area that responds specifically to optic flow, revealed by fMRI. Nat Neurosci 3:1322-8 
Mosher J, Leahy R, Lewis P (1999) EEG and MEG: forward solutions for inverse methods. IEEE Trans Biomed Eng 46:245-59

Nakamura H (2003) Human V5 demonstrated by magnetoencephalography using random dot kinematograms of different coherence levels. Neurosci Res 46:423-33

Noesselt T, Rieger J, Schoenfeld M, Kanowski M, Hinrichs H, Heinze H, Driver J (2007) Audiovisual temporal correspondence modulates human multisensory superior temporal sulcus plus primary sensory cortices. J Neurosci 27:11431-41

Noguchi Y, Kaneoke Y, Kakigi R, Tanabe H, Sadato N (2005) Role of the superior temporal region in human visual motion perception. Cereb Cortex 15:1592-601

Oostenveld R, Fries P, Maris E, Schoffelen J (2011) FieldTrip: Open source software for advanced analysis of MEG, EEG, and invasive electrophysiological data. Comput Intell Neurosci 2011:156869

Overath T, Kumar S, Stewart L, Kriegstein K von, Cusack R, Rees A, Griffiths T (2010) Cortical mechanisms for the segregation and representation of acoustic textures. J Neurosci 30:2070-6

Pascual-Leone A, Hamilton R (2001) The metamodal organization of the brain. Prog Brain Res 134:427-45

Pelli D (1997) The VideoToolbox software for visual psychophysics: transforming numbers into movies. Spat Vis 10:437-42

Poirier C, Collignon O, Devolder A, Renier L, Vanlierde A, Tranduy D, Scheiber C (2005) Specific activation of the V5 brain area by auditory motion processing: an fMRI study. Brain Res Cogn Brain Res 25:650-8

Poirier C, Collignon O, Scheiber C, Renier L, Vanlierde A, Tranduy D, Veraart C, Volder A De (2006) Auditory motion perception activates visual motion areas in early blind subjects. Neuroimage 31:279-85

Powers A, Hevey M, Wallace M (2012) Neural correlates of multisensory perceptual learning. J Neurosci 32:6263-74

Proulx M, Brown D, Pasqualotto A, Meijer P (2012) Multisensory perceptual learning and sensory substitution. Neurosci Biobehav Rev

Rauschecker J, Tian B (2000) Mechanisms and streams for processing of "what" and "where" in auditory cortex. Proc Natl Acad Sci USA 97:11800-6

Renier L, Volder A De, Rauschecker J (2013) Cortical plasticity and preserved function in early blindness. Neurosci Biobehav Rev:1-11

Ricciardi E, Bonino D, Pellegrini S, Pietrini P (2013) Mind the blind brain to understand the sighted one! Is there a supramodal cortical functional architecture? Neurosci Biobehav Rev 
Ricciardi E, Pietrini P (2011) New light from the dark: what blindness can teach us about brain function. Curr Opin Neurol 24:357-63

Ricciardi E, Vanello N, Sani L, Gentili C, Scilingo E, Landini L, Guazzelli M, Bicchi A, Haxby J, Pietrini P (2007) The effect of visual experience on the development of functional architecture in hMT+. Cereb Cortex 17:2933-9

Roe A, Pallas S, Hahm J, Sur M (1990) A map of visual space induced in primary auditory cortex. Science (80-. ). 250:818-20

Romanski L (2004) Domain specificity in the primate prefrontal cortex. Cogn Affect Behav Neurosci 4:421-9

Romanski L (2007) Representation and integration of auditory and visual stimuli in the primate ventral lateral prefrontal cortex. Cereb Cortex 17:61-9

Romanski L, Hwang J (2012) Timing of audiovisual inputs to the prefrontal cortex and multisensory integration. Neuroscience 214:36-48

Romanski L, Tian B, Fritz J, Mishkin M, Goldman-Rakic P, Rauschecker J (1999) Dual streams of auditory afferents target multiple domains in the primate prefrontal cortex. Nat Neurosci 2:1131-6

Rousseeuw P, Leroy A (1987) Robust regression and outlier detection. New York: Wiley.

Saldern S von, Noppeney U (2013) Sensory and striatal areas integrate auditory and visual signals into behavioral benefits during motion discrimination. J Neurosci 33:8841-9

Sasaki Y, Nanez J, Watanabe T (2010) Advances in visual perceptual learning and plasticity. Nat Rev Neurosci 11:53-60

Savitzky A, Golay M (1964) Smoothing and differentiation of data by simplified least squares procedures. Anal Chem 36:1627-39

Saygin A (2007) Superior temporal and premotor brain areas necessary for biological motion perception. Brain 130:2452-61

Schafer R (2011) What is a Savitzky-Golay filter? IEEE Signal Process Mag 28:111-7

Scheef L, Boecker H, Daamen M, Fehse U, Landsberg M, Granath D, Mechling H, Effenberg A (2009) Multimodal motion processing in area V5/MT: evidence from an artificial class of audio-visual events. Brain Res 1252:94-104

Schroeder C, Foxe J (2005) Multisensory contributions to low-level, “unisensory” processing. Curr Opin Neurobiol 15:454-8

Schwartz J, Berthommier F, Savariaux C (2004) Seeing to hear better: evidence for early audio-visual interactions in speech identification. Cognition 93:B69-78

Seitz A, Kim R, Shams L (2006) Sound facilitates visual learning. Curr Biol 16:1422-7 
Seitz A, Kim R, Wassenhove V van, Shams L (2007) Simultaneous and independent acquisition of multisensory and unisensory associations. Perception 36:1445-53

Seitz A, Watanabe T (2005) A unified model for perceptual learning. Trends Cogn Sci 9:329_ 34

Shams L, Seitz AR (2008) Benefits of multisensory learning. Trends Cogn Sci 12:411-417

Song C, Kanai R, Fleming S, Weil R, Schwarzkopf D, Rees G (2011) Relating interindividual differences in metacognitive performance on different perceptual tasks. Conscious Cogn 20:1787-92

Stein B, Meredith M (1993) The merging of the senses. Cambridge, MA, US: MIT Press.

Striem-Amit E, Dakwar O, Reich L, Amedi A (2012) The large-scale organization of "visual" streams emerges without visual experience. Cereb Cortex 22:1698-709

Sugihara T, Diltz M, Averbeck B, Romanski L (2006) Integration of auditory and visual communication information in the primate ventrolateral prefrontal cortex. J Neurosci 26:11138-47

Talsma D, Senkowski D, Soto-Faraco S, Woldorff M (2010) The multifaceted interplay between attention and multisensory integration. Trends Cogn Sci 14:400-10

Taulu S, Simola J (2006) Spatiotemporal signal space separation method for rejecting nearby interference in MEG measurements. Phys Med Biol 51:1759-68

Tootell R, Reppas J, Kwong K, Malach R, Born R, Brady T, Rosen B, Belliveau J (1995) Functional analysis of human MT and related visual cortical areas using magnetic resonance imaging. J Neurosci 15:3215-30

Ungerleider L, Mishkin M (1982) Two cortical visual systems. D. Ingle, M. Goodale, \& J. Mansfield, eds. Cambridge, MA, US: MIT Press.

Voss P, Zatorre R (2012) Organization and reorganization of sensory-deprived cortex. Curr Biol 22:R168-73

Watkins K, Shakespeare T, O’Donoghue M, Alexander I, Ragge N, Cowey A, Bridge H (2013) Early auditory processing in area V5/MT+ of the congenitally blind brain. J Neurosci 33:18242-6

Watson J, Myers R, Frackowiak R, Hajnal J, Woods R, Mazziotta J, Shipp S, Zeki S (1993) Area V5 of the human brain: evidence from a combined study using Positron Emission Tomography and Magnetic Resonance Imaging. Cereb Cortex 3:79-94

Weisberg S (2005) Outliers and influence. In Applied Linear Regression Hoboken: Wiley, p. 194-210.

Werner S, Noppeney U (2010) Distinct functional contributions of primary sensory and association areas to audiovisual integration in object categorization. J Neurosci 30:2662-75 
Wichmann F, Hill N (2001) The psychometric function: I. Fitting, sampling, and goodness of fit. Percept Psychophys 63:1293-313

Wierzchoń M, Asanowicz D, Paulewicz B, Cleeremans A (2012) Subjective measures of consciousness in artificial grammar learning task. Conscious Cogn 21:1141-53

Zeki S, Watson J, Frackowiak R (1993) Going beyond the information given: the relation of illusory visual motion to brain activity. Proc Biol Sci 252:215-22 